\title{
Evaluation of 0D Stochastic Reactor Modelling for a Diesel Engine Application
}

\author{
Aleksandrs Korsunovs' ${ }^{1}$, Felician Campean', Gaurav Pant ${ }^{1}$, Oscar Garcia-Afonso ${ }^{2}$, \\ Efe Tunc ${ }^{2}$ \\ ${ }^{1}$ Automotive Research Centre, University of Bradford, UK \\ ${ }^{2}$ Jaguar Land Rover, UK
}

\begin{abstract}
Prediction of engine-out emissions with high fidelity from in-cylinder combustion simulations is still a significant challenge early in the engine development process. This paper contributes to this fast evolving body of knowledge by focusing on the evaluation of NOx emissions predictions capability of a Probability Density Function (PDF) based Stochastic Reactor Engine Models (SRM), for a Diesel engine. The research implements a systematic approach to the study of the SRM engine model performance, based on a detailed space-filling design of experiments based sensitivity analysis of both external and internal parameters, evaluating their effects on the accuracy in matching physical measurements of in-cylinder conditions, and NOx emissions output. The approach proposed in this paper introduces an automatic SRM model calibration methodology across the engine operating envelope, based on a multi-objective optimization approach. This aims to exploit opportunities for internal SRM parameters tuning to achieve good overall modelling performance as a trade-off between physical in-cylinder measurements accuracy and the output NOx emissions predictions error. The results from the case study provide a valuable insight into the effectiveness of the SRM model, showing good capability for NOx emissions prediction and trends, while pointing out the critical sensitivity to the external input parameters and modelling conditions.
\end{abstract}

Keywords: Thermodynamic models, Stochastic Reaction Model, PDF, NOx emissions, OLH Experiments.

\section{Introduction}

Computer-assisted virtual engineering methods are playing an increasing role in engine systems development and validation, in particular for the early stages of the product development cycle. This is driven by the economic and development time benefits offered by computer modelling techniques, compared with the traditional development processes that involve validation testing on physical prototypes. Availability of simulation based virtual prototypes early in the engine development process, can lead to significant enhancement in the efficiency of the development process, in terms of both time and cost. 
However, there are still challenges with the development and validation of CAE system models for Internal Combustion Engine that can be used with confidence early in an engine programme to evaluate economy and emissions performance. A main challenge stems from the fact that high fidelity models (e.g. 3-dimensional CFD models) involve computationally intensive processes that can take considerable amount of time to converge. This is incompatible with the practical / industry requirements for virtual prototyping, demanding capability for real time simulation of the entire airpath through the engine, such that driveability and emissions performance (including transient performance) can be evaluated against an increasing number of drive cycles (including real world drive cycles). Addressing this challenge requires an integrated multi-physics approach to the system modelling task with variable levels of fidelity depending upon the development stage and the specific purpose of the analysis.

The work presented in this paper focusses on the validation of a CAE engine modelling approach for prediction of engine out emissions, in particular NOx emissions from a Diesel engine. The work is underpinned by recent developments and use of thermodynamic models for in-cylinder combustion. Such thermodynamic models are advanced in terms of chemical and in-cylinder conditions modelling, and have demonstrated good prediction capabilities for emissions, with relatively fast computation speeds in comparison to 3D CFD modelling. Prominent approaches and capability now available include the commercially available package developed by CMCL Innovations, referred to herein as CMCL SRM, which has been tested and validated in applications including diesel engine entire speed-load domain simulation [1], diesel engine optimisation [2], and simulation of HCCI engines [3]-[5]. Building on collaborative work with several European Universities, Loge AB have provided an alternative stochastic reactor model, which achieves simulation times reduction [6], and allows for in-depth study of the mixing time profiles impact and two-zone thermodynamic models application [7], use of Eulerian Field Probability Density Function Methods [8] and tabulation strategy [9].

While thermodynamic models offer promising capability for modelling engine out emissions with an acceptable compromise between fidelity and computation time, there are further challenges with implementation and use of these tools in industry practice. One such challenge is the setting up and validation of a thermodynamic model for a specific engine or engine application. A stochastic reactor engine model requires a large number of input parameters and boundary conditions to be specified, based on the specific engine modelling task and in relation to physical measurements from specific engine tests, which are in turn affected by uncertainties. This tuning task can become quite involved and time consuming, given that a comprehensive characterisation of engine performance across the entire operating space for the target application is normally required. Validation of performance of the SRM engine for predicting emissions with acceptable fidelity within this envelope is also required.

The research presented in this paper was specifically aimed at addressing this challenge, and to contribute to this fast evolving body of knowledge with important methodological developments enabling a robust calibration and validation of an SRM engine model. The work is based on a Diesel engine case study, and a commercially available software 
package that offer thermodynamic combustion modelling solutions CMCL Kinetics and SRM Engine Suite. The CMCL SRM offers the possibility to build Probability Density Function based Stochastic Reactor Thermodynamic models that allow detailed chemical composition prediction.

This research implements a systematic approach to the study of the SRM engine model performance, based on a detailed space-filling design of experiments based sensitivity analysis of both external and internal parameters, evaluating their effects on the accuracy in matching physical measurements of in-cylinder conditions, and NOx emissions output. The approach proposed in this paper introduces an automatic SRM model calibration methodology across the engine operating envelope, based on a multi-objective optimization approach. This aims to exploit opportunities for internal SRM parameters tuning to achieve good overall modelling performance as a trade-off between physical incylinder measurements accuracy and the output NOx emissions predictions error.

While the main research contribution of the work is in the methodological developments for the efficient SRM model calibration and validation, the results from the case study provide a valuable insight into the effectiveness of the SRM model to predict emissions for Diesel engine applications early in system design and calibration development.

The organization of the paper is as follows: section 2 of the paper provides an overview of related work on thermodynamic engine models and the used of probability density functions (pdf) with thermodynamic models. Section 3 outlines the research methodology employed in this research. It includes an outline of the engine case study, development of the SRM model, the base procedure for the SRM engine model calibration, and the outline of the DoE methodology employed for the SRM engine sensitivity study. Section 4 covers the sensitivity study results with a detailed analysis and discussion of the results. Section 5 presents the methodology for SRM engine model calibration across the relevant engine calibration envelope, based on a multi- objective optimization approach. The paper ends up with a discussion of methodology and results, and a summary of the main conclusions and contributions.

\section{Review of Related Literature on Thermodynamic Engine Models}

\section{Thermodynamic Engine Models}

Thermodynamic engine models, underpinned by the laws of conservation of mass and energy, and mean value models that offer significant computational advantages as they do not account for information about spatial fields of various physical parameters distribution, such as pressure or temperature. However, this attracts prediction accuracy drawbacks, as well as limited state information available. Thermodynamic models are well established and are frequently employed for engine power prediction, efficiency, fuel consumption, losses in the engine and emissions [10].

Single-zone thermodynamic models treat the whole system as a single volume and assume homogeneity of physical parameters throughout the simulation. Such models are fast and 
can be useful for parameter studies and general prediction, but provide lower fidelity for the in-cylinder combustion modelling. Reasonable matching of the single zone simulation models against experimental data is only achievable when the air/fuel mixture and temperature distribution in the cylinder is close to uniform [2]. Dibble et al [11], Arsie et al [12] and Ngwaka et al [15] have given examples of single-zone model applications where they have been able to prove reasonable prediction performance for the start of the combustion, as well as the peak cylinder pressure, indicated efficiency and the NOx emissions.

Multi-zone thermodynamic models split the open system volume in zones. Each zone has different physical properties, which are uniform within the zone. This approach must not be confused with the multi-dimensional models, because the zones do not provide any spatial representation of the system domain, they only represent parts of the system that are likely to have different properties. The prediction capabilities of multi-zone models can, in general, be expected to be higher compared to single-zone models. In particular, this becomes obvious when the engine running conditions create heterogeneous distribution of physical properties within the cylinder, in which case multi-zone models give significant improvement of the pressure profile prediction and allow to estimate $\mathrm{CO}$ and HC emissions [11], [12]. However, for some applications, such as study of the natural gas HCCI engine presented in [13], focused on NOx estimation, the use of multi-zone models did not provide a significant improvement over the single-zone modelling approach. Multi-zone models have been shown to be instrumental for providing more accurate emissions prediction [14]-[17], in particular $\mathrm{CO}, \mathrm{CH}$ and $\mathrm{Pn}$, as particular parts of the cylinder volume (such as cylinder wall boundary layer or piston crevice volume) play an important role in formation of these.

Most of the modern thermodynamic models include sub-models for various essential physical phenomena, such as spray atomisation, liquid phase penetration, evaporation, diffusive combustion, auto-ignition, heat transfer and turbulence [18].

More advanced quasi-dimensional thermodynamic models have been recently developed. Such models take account of some of the geometric features and may include the shape of the diesel spray cone [19] and flame front propagation [20] to enhance the modelling fidelity.

\section{Use of Probability Density Function in Thermodynamic Models}

In order to account for the stochastic nature of the combustion process, a probability density function transport equation is introduced into the model. PDF-based Stochastic Reactor Models are still zero-dimensional, which implies that that the variables are not dependent on space. However, it is important to note that the model does not assume homogeneity throughout the space, but statistical homogeneity of the space. In other words, the PDF transport equation is the same throughout the spatial domain.

In order to appropriately represent the in-cylinder variability, so-called "stochastic particles" are introduced to represent the statistical variability of the information about the 
mass fractions of the chemical species involved in the process, as well as the temperature, hence the term - Stochastic Reactor Model (SRM).

PDF approaches have attracted attention from both the academic research and the industrial application domains, as they offer satisfactory prediction level of the in-cylinder mixture composition and temperature, requiring relatively low computational and time expense. Significant work has been reported on the use of the PDF-SRM approach for Compression Ignition (diesel) engines in [1], [18], [21] - [25], gasoline direct injection engine in [25] [27] and the Homogeneous Charge Compression Ignition engines in [4], [5], [28]-[32]. Within the field of Compression Ignition engines, the SRM approach was shown to be able to predict the in-cylinder pressure trace and heat release rate fairly well [21], [22]. Similar good performance has been reported for predicting NOx emissions [1], [18]. However, predicting $\mathrm{HC}, \mathrm{CO}$ and $\mathrm{Pn}$ is a still challenge [23], [24].

Information about SRM applications for Gasoline Spark Ignition engines simulation is limited. SRM has been successfully used for the validation of the in-cylinder conditions against the experimental data and knock combustion detection [26]. In relation to emissions, while it has been shown that the model can generally predict very well the trends for the $\mathrm{HC}, \mathrm{CO}, \mathrm{CH}, \mathrm{NOx}$ and $\mathrm{Pn}$, the fidelity of the predictions is still being questioned [25], [27].

Other SRM applications include the HCCI engines study, which illustrates very good correlation with the experimental data in terms of the in-cylinder conditions, but has limitations in emissions prediction, when validated against the experimental data [4], [28].

From a practical application perspective, an additional challenge that needs to be considered is the suitability of a single SRM model for the entire operating space of an engine and a particular application of that engine. Previously, SRM has been used to model light duty [33],[34] and heavy duty [33],[35] engines operating domains, reporting good performance throughout. However, it is important to note that neither of these sources considered more than two injections per cycle, which is now common in light duty engine applications, as the combustion and control strategies are becoming more complicated, and the NOx model performance was not evaluated in all studies.

\section{Research Methodology}

The work in this paper is concerned with the evaluation of the capability of CMCL SRM environment for modelling of Diesel engine combustion, with a particular focus on NOx emissions prediction. The review of the literature has identified SRM as capable of modelling in-cylinder conditions (pressure trace and heat release) [21] \& [22], and predict fairly well NOx emissions [1] \& [18].

This work is based on a Diesel engine case study, with the following research aims defined in relation to the SRM engine simulation: 
1. to evaluate the capability to model the combustion within the Diesel engine studied across the engine speed / load calibration envelope; and

2. to evaluate the NOx prediction capability of the SRM model.

Addressing the first research aim requires the development of a methodology for calibration and validation of the SRM engine model to perform with acceptable fidelity across the engine operating range included in the test. This requires a detailed sensitivity study of the SRM engine model outputs (in-cylinder conditions and NOx predictions) in relation to both the model inputs (referred to in the following as external SRM parameters) and internal SRM model parameters.

This section outlines the experimental and modelling setup including the Diesel engine case study and the SRM engine model development, and the detailed planning of the sensitivity study using a design of experiments methodology.

\section{Experimental and modelling set up}

The experimental and modelling set up for this work is depicted in Figure 1. The reference engine case study is a 2.0 litre Diesel engine (specification outlined in table 1), for which extensive experimental data was available from dynamometer calibration testing.

Figure 1 illustrates the methodology for SRM modelling and validation using system diagrams. Engine test data was collected from steady state hot calibration dynamometer tests conducted at a set of 23 pre-defined engine speed - load set points, illustrated in Figure 2. At each of the 23 engine speed-load point tested, records of in-cylinder pressure traces over a number of cycles were available, along with emissions measurements taken at turbine outlet. An SRM model of the engine was developed using the same inputs and conditions as the physical engine tests. The parameters associated with the engine operation are listed as Inputs in Figure 1, including specific parameters required for the SRM models (relating to the engine and the input flows) shown in italics. The SRM model uses a range of internal parameters (also shown in Figure 1), which can be tuned to improve the accuracy of the modelling. The in-cylinder pressure profiles reconstructed from physical measurements during testing are used to evaluate, calibrate and validate the SRM engine model. The performance of the SRM engine model also evaluated in relation to correlation of the output NOx predictions compared with the actual measurements from the engine tests.

Table 1. Experimental Engine Basic Information

\begin{tabular}{|c|c|}
\hline Parameter & Value \\
\hline Bore & $83 \mathrm{~mm}$ \\
\hline Stroke & $92.35 \mathrm{~mm}$ \\
\hline Connecting Rod Length & $140 \mathrm{~mm}$ \\
\hline Compression Ratio & 15.5 \\
\hline Emissions Standard & Euro $6 \mathrm{c}$ \\
\hline
\end{tabular}




\begin{tabular}{|c|c|}
\hline Peak Power & 130 kW @ 4000 RPM \\
\hline Peak Torque & 430 Nm @ 1750-2500 RPM \\
\hline
\end{tabular}

\begin{tabular}{|l|l|l||}
\hline - Engine Speed & \\
Torque & Geometry
\end{tabular}

Figure 1: Experimental and Modelling Set up.

\section{SRM Model Development}

An SRM engine model was constructed for the case study Diesel engine in the CMCL SRM modelling environment, with the general set-up shown in Table 2. The parameters required for the development of the SRM engine model, summarized in Figure 1, include 'external' / input parameters relating to both the engine test parameters (e.g. fuel, ambient conditions) and the speed-load specific parameters (calibration state variables) used in the experimental engine tests. Table 3 summarizes the SRM required modelling parameters.

Table 2. SRM Model Basic Information

\begin{tabular}{|c|c|}
\hline Parameter & Value \\
\hline Simulation Step Size & 0.2 CAD \\
\hline Number of Stochastic Particles & 100 \\
\hline Non-Equiweighting Factor & 15 \\
\hline Solver & Solver-2 (BDF) \\
\hline Relative Tolerance & $1 \mathrm{e}-5$ \\
\hline Absolute Tolerance & \\
\hline
\end{tabular}




\begin{tabular}{|l|c|}
\hline Surrogate Fuel Composition & $\begin{array}{c}80 \% \mathrm{n} \text {-Heptane } \\
20 \% \mathrm{i} \text {-Octane }\end{array}$ \\
\hline
\end{tabular}

Table 3: SRM External Input Parameters.

\begin{tabular}{|c|c|}
\hline $\begin{array}{l}\text { Engine Geometry } \\
{[\text { constant for every engine }]}\end{array}$ & $\begin{array}{l}\text { Simplified cylinder geometry, } \\
\text { including: } \\
\text { - } \quad \text { Bore and stroke } \\
\text { - } \quad \text { Length of the connecting rod } \\
\text { - } \\
\text { - } \\
\text { - } \\
\text { Wrist pin offset } \\
\text { Crevice dimensions }\end{array}$ \\
\hline $\begin{array}{l}\text { Intake Mixture Static } \\
{[\text { Constant for }} \\
\text { engine/location] }\end{array}$ & $\begin{array}{ll} & \text { Air chemical composition } \\
\text { - } & \text { EGR composition settings }\end{array}$ \\
\hline $\begin{array}{l}\text { Intake Mixture Dynamic } \\
\text { [Speed-load point specific] }\end{array}$ & $\begin{array}{ll}\text { - } & \text { Intake mixture temperature at } \\
\text { IVC } \\
\text { - } \\
\text { Intake mixture pressure at IVC } \\
\text { EGR mass fraction }\end{array}$ \\
\hline $\begin{array}{l}\text { Fuel System Static } \\
\text { [Constant for every engine and } \\
\text { fuel] }\end{array}$ & 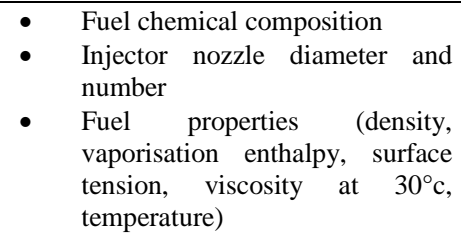 \\
\hline $\begin{array}{l}\text { Fuel System Dynamic } \\
\text { [Speed-load point specific] }\end{array}$ & $\begin{array}{ll}- & \text { Injection pressure } \\
- & \text { Injection rate profile }\end{array}$ \\
\hline $\begin{array}{l}\text { In-cylinder Wall Temperatures } \\
\text { [Speed-load point specific] }\end{array}$ & $\begin{array}{l}\text { - } \quad \text { Piston, cylinder head, cylinder } \\
\text { liner wall temperatures }\end{array}$ \\
\hline
\end{tabular}

A challenge with setting up the SRM model stems from the fact that some of the external parameters are not directly available from measurements from the normal calibration engine tests. For example, the internal EGR (iEGR) is an important input to the SRM combustion model; however, this cannot be measured experimentally. For the purpose of this work, surrogate data from a validated 1D simulation model (illustrated in Figure 3) developed for the specific engine / application was available, and was used to provide estimates for the iEGR input to the SRM engine model. Other physical variables that present a challenge in terms of experimental measurement are the in-cylinder mixture temperature at the inlet valve closing (IVC) and the in-cylinder wall temperatures. For the in-cylinder mixture temperature, the closest measurement available from the test data was the inlet port temperature, which does not exactly represent the required input. For the purpose of this work, an airpath 1D simulation model of the engine was used to estimate these temperature inputs requirements. The injection rate profiles for each of the speedload points were obtained from an injector simulation model available for this purpose. 


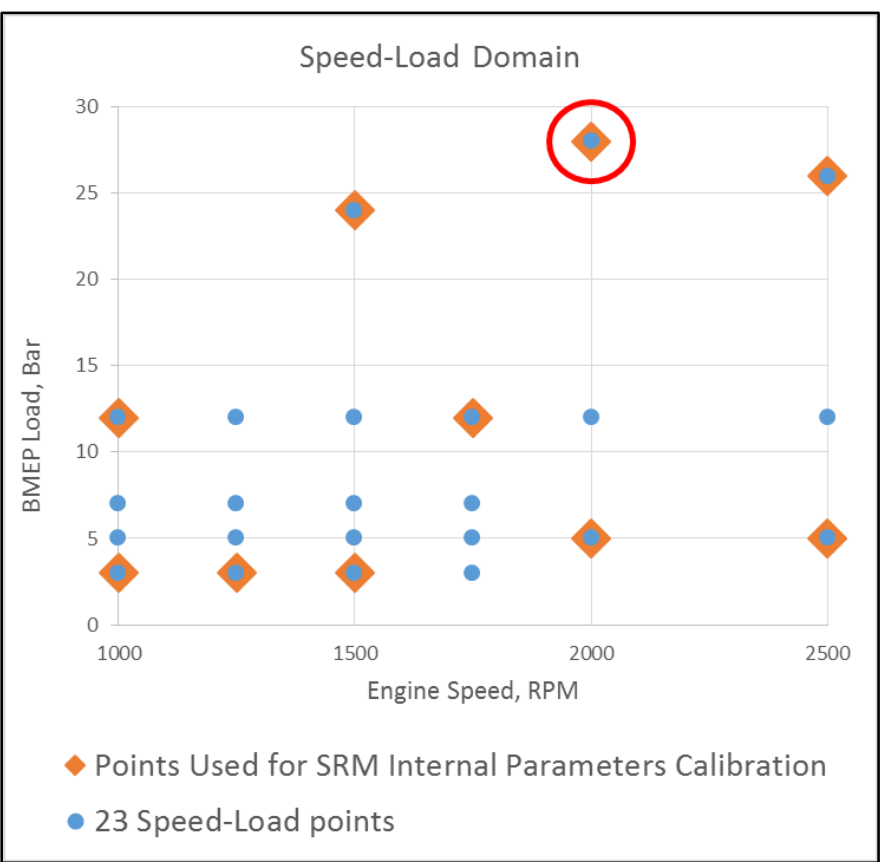

Figure 2: Experimental engine test points available for the study and the SRM calibration points (highlighted points)

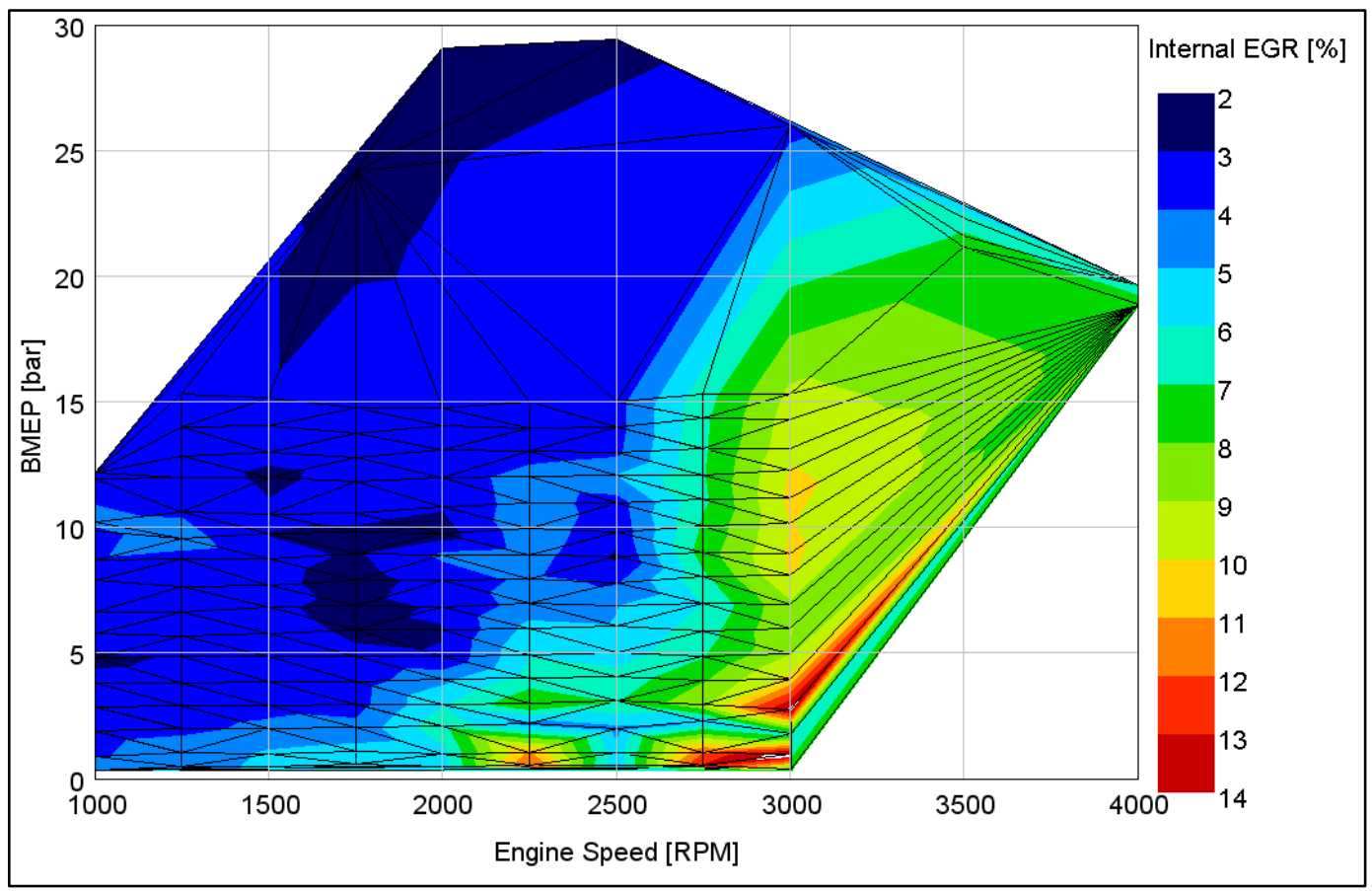

Figure 3: Internal EGR (iEGR) Map from validated 1D Model.

Aside from the engine external input parameters, the SRM model uses a set of internal parameters, associated with the thermodynamic sub-models, are discussed in the following sub-sections. 
Sauter Mean Diameter (SMD)

The SMD variable is related to the assumed diameter of a droplet that has the same surface to volume ratio as the whole spray. In order to obtain the SMD at any point of cycle, the SRM employs empirical equation that was derived by Elkotb [36]:

$$
D_{S M D}=A v^{B} \sigma^{C} \rho_{f}^{D} \rho_{a}^{E} \Delta P^{F}
$$

Where: A-F are empirical parameters; $v$ - fuel viscosity; $\sigma$ - surface tension; $\rho_{\mathrm{f}}$ - fuel density; $\rho_{\mathrm{a}}$ - in-cylinder air/fuel mixture density; $\Delta \mathrm{P}$ - pressure difference between the fuel rail and the ambient pressure.

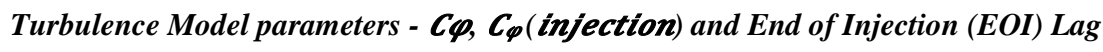

$C \varphi$, defined in equation 2 [37], is a mechanical-to-scalar timescale ratio used to adjusts the intensity of turbulence in the thermodynamic model.

$$
\frac{1}{\tau_{m}}=\frac{C_{\varphi}}{2 \tau}
$$

The intensity in this case is usually quantified by scalar mixing rate, which is defined in terms of mixing time $\left(\tau_{\mathrm{m}}\right)$. Adjusting $\mathrm{C} \varphi$ allows tuning of the conversion from the turbulence timescale $(\tau)$ to the mixing time. There are two constants for this purpose in the CMCL SRM model:

- $\mathrm{C} \varphi($ injection) accounts for the conversion during the injection events;

- $\mathrm{C} \varphi$ accounts for the conversion through the rest of the cycle.

The EOI lag parameter allows adjustment for the amount of crankshaft-angle degrees for which the $\mathrm{C} \varphi$ (injection) is still applied after the end of injection. This is important in order to account for the turbulence induced by the combustion itself.

\section{Injection model - Alpha}

The Alpha calibration parameter controls the fuel distribution between the stochastic particles, i.e. quantifies the charge stratification extent in the cylinder after the injection. Higher Alpha values point to an inhomogeneous distribution, while lower Alpha values means a more homogeneous distribution. The mathematical representation is shown in equation 3 [37].

$$
F^{(i)}=e^{\left(-\alpha\left(\frac{\sum_{i=1}^{i} m^{i}}{\sum_{i=1}^{N p a r} m^{i}}\right)\right)^{2}}
$$

where $\mathbf{F}$ is the Probability Distribution Function, $\mathbf{m}$ is the evaporated mass, $\boldsymbol{\alpha}$ is Alpha and $\mathbf{i}$ is the particle number. 
Evaporation model Constant - Lambda

The lambda constant within the evaporation model allows accelerating or slowing down the evaporation rate of the fuel. Higher Lambda values facilitate the evaporation, and, in turn, lower values slow it down [37].

\section{Heat Transfer Model - Woschni Constants C1 and C2}

The Woschni constants $\mathrm{C} 1$ and $\mathrm{C} 2$ are necessary to account for the changes in the gas velocity throughout the engine cycle. The constant $\mathrm{C} 1$ adjusts the effect of the mean piston speed on the heat transfer, whereas the constant $\mathrm{C} 2$ accounts for the representation of the combustion effect on the gas velocity in the cylinder. The full Woschni correlation can be seen in equation 4 [38]:

$$
\begin{aligned}
h=129.9 B^{-0.2} & p^{0.8} T_{g}^{-0.53}\left[C_{1} V_{p}\right. \\
& \left.+C_{2} \frac{V_{s} T_{r}}{p_{r} V_{r}}\left(p-p_{m}\right)\right]^{0.8}
\end{aligned}
$$

Where $\mathbf{h}$ is heat transfer coefficient $\left(\frac{W}{m^{2} K}\right), \mathbf{p}$ is in-cylinder pressure $(\mathrm{Pa}), \mathbf{T g}$ is in-cylinder gas temperature, $\mathbf{B}$ is bore $(\mathrm{m}), \mathbf{V} \mathbf{p}$ is mean piston speed $(\mathrm{m} / \mathrm{s}), \mathbf{V s}$ is swept volume $\left(\mathrm{m}^{3}\right)$, $\mathbf{p}_{\mathbf{m}}$ is mean in-cylinder pressure $(\mathrm{Pa})$, and all the values denoted with " $\mathrm{r}$ " index are corresponding to conditions at a reference state (such as inlet valve close or beginning of the combustion).

Given that the Woschni C1 constant does not take combustion into account, a good alignment of the simulated in-cylinder pressure curve at the compression stroke, before the first pilot injection, with the experimental trace provides a reasonable indication that the heat transfer model accounts for the heat losses correctly.

\section{Procedure for SRM Engine Model Calibration}

Once the SRM engine model is developed it is important to validate that the behaviour of the SRM model matches that of the engine modelled. A good SRM model is expected to match the experimental in-cylinder pressure traces with an acceptable error, and that the predictions for the output emissions match the trends and values observed experimentally with reasonable accuracy. Further analysis of the SRM model performance can be done based on the comparison of the Apparent Heat Release Rate (AHRR) profiles, which can be derived from the pressure profiles directly, using the equation 5 [39]:

$$
\frac{d Q}{d \theta}=\frac{\gamma}{\gamma-1} P \frac{d V}{d \theta}+\frac{1}{\gamma-1} V \frac{d P}{d \theta}
$$

where $\mathbf{Q}$ is the heat released $(\mathrm{J}), \boldsymbol{\Theta}$ is CAD, $\boldsymbol{\gamma}$ is the ratio of specific heat capacities $\left(\frac{C p}{C v}\right), \mathbf{P}$ is the in-cylinder pressure $(\mathrm{Pa})$ and $\mathbf{V}$ is the in-cylinder volume $\left(\mathrm{m}^{3}\right)$. 
The tuning of an SRM model (i.e. identification of a set of optimal values of the SRM model parameters such that the errors from the experimental data are minimised) can be a very complex and time-consuming task for an engine development team, but it is an essential and critical step if an efficient model is to be achieved. Some of the challenges associated with this task stem from the fact that engine experimental data is in turn affected by significant random variation. For example, the experimental in-cylinder pressure traces show cycle to cycle variability, as illustrated in Figure 4, where several in-cylinder pressure traces are illustrated. Good practice in the development of thermodynamic engine models [40] suggests that an actual cycle (rather than a synthetic arithmetic average) should be taken as reference for calibration / validation of the model, such that the appropriate thermodynamic behaviour is correctly modelled. This raises the need for developing an appropriate procedure for selecting a representative median actual cycle from the engine collected data. This becomes even more challenging for situations where there are multiple injection events (e.g. pilot and main injection) - which is the case illustrated in Figure 4, as selection of the median cycle based solely on the peak pressure or location (in CAD) based on the methodology described in [27], does not necessarily guarantee a global median cycle. Therefore, a procedure has been developed for the selection of the median cycle, which will be described in the following section.

\section{Procedure for median cycle selection}

Given the multi-modal behavior of the in-cylinder pressure traces illustrated in Figure 4, the proposed procedure for selection of a median cycle is based dual ranking of in-cylinder pressure traces based on zonal criteria. The first zone considered is for the CAD range between Start of Injection (SOI) and End of Injection (EOI). The pilot injection ignition takes place at this stage, which affects the combustion process; given that the SOI and EOI stay fixed throughout the test for a particular speed-load point, this makes it possible to compare the calculated quantities in a more consistent manner. The second zone is the main pressure peak, where the in-cylinder pressure traces can be ranked according to the maximum pressure.

The procedure for selection of the median cycle consists of the following steps:

1. The area below each of the pressure curves is calculated (Equation 6) within the range of Start of Injection (SOI) and End of Injection (EOI).

$$
A=\int_{\text {SOI }}^{E O I} f(x) d x
$$

where $\mathrm{f}$ is the in-cylinder pressure profile and $\mathrm{x}$ is the CAD step.

2. The pressure trace with the lowest area is chosen as a base "minimum" curve, $A_{\min }$;

3. The sum of squared errors (SSE) is calculated on a CAD basis between the SOI and the EOI for each of the pressure curves against the chosen minimum curve, i.e.: 


$$
S S E_{i}=\sum_{k=S O I}^{E O I}\left(y_{i}(k)-a(k)\right)^{2}
$$

where $\boldsymbol{y}_{\boldsymbol{i}}$ is the current pressure profile, $\boldsymbol{a}$ is the base pressure profile corresponding to the minimum area $A_{\min }$, and $\boldsymbol{k}$ is the CAD step.

4. The pressure traces are ranked according to their SSE, and the median cycle $\boldsymbol{C}_{\text {SSEm }}$ is chosen as reference.

5. The pressure curves are ranked according to the recorded in-cylinder peak pressure $\left(\boldsymbol{P P}_{\boldsymbol{i}}\right)$, and a median cycle is selected based on peak pressure, $\boldsymbol{C}_{\boldsymbol{P P m} \boldsymbol{}}$.

6. The cycle selected as the median is the one that is closest to the 2 selected median cycles; mathematically, this can be articulated as a distance minimisation problem, i.e.

$$
\begin{array}{cl}
\min \{d\} ; & d_{i}=\left|R_{S S E}\left(C_{i}\right)-R_{m}\right| \\
& +\left|R_{P P}\left(C_{i}\right)-R_{m}\right|
\end{array}
$$

Where $R_{S S E}\left(C_{i}\right)$ denotes the rank of the cycle $C_{i}$ based on the calculated SSE between SOI and EOI, and $R_{P P}\left(C_{i}\right)$ denotes the rank of the cycle $C_{i}$ based on the peak pressure. If two cycles yield the same minimum distance, then the cycle with the smallest peak pressure error $e_{i}$, calculated as in equation 9 , will be chosen.

$$
e_{i}=\left|P P_{i}-P P_{m}\right|
$$

Figure 4 illustrates the selected median cycle (the bold dashed line) for a particular engine speed / load test point.

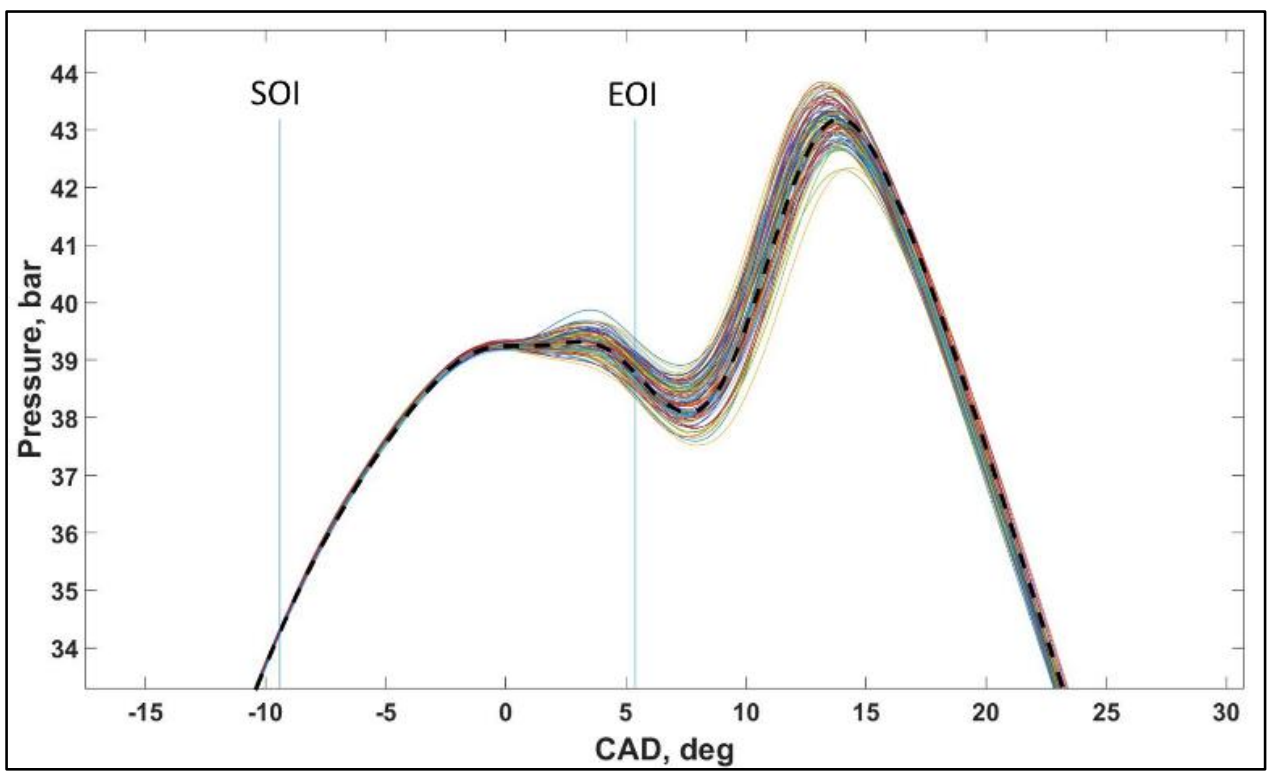

Figure 4: In-cylinder pressure traces and median cycle selection. 
The median cycle selected using the methodology described above was used to support the calibration and validation of the SRM engine model.

\section{Design of Experiments Methodology for SRM Sensitivity Study}

The "calibration" of an SRM engine model relates to the choice of internal model parameters such that the modelling error is minimised. In practice, we are also interested in enhancing the prediction power of the model, so the study should also include the effect of the choice of the model parameters on the predictions for the outputs, specifically emissions.

It is therefore of interest to study the sensitivity of the SRM modelling error (in relation to the median cycle in-cylinder pressure trace) and the emissions output prediction error (as relative error to the NOx measured on test), in relation to:

(i) uncertainty in the external input parameters (shown in Table 3); and

(ii) the internal SRM model parameters.

The approach taken in this research was to employ a structured Design of Experiments (DoE) methodology for the SRM model sensitivity study. Given the computation expense associated with running the SRM engine model (commonly taking 100 - 600 seconds per cycle), a DoE approach is needed to enable an efficient strategy. For this purpose, the exploration-based sequential DoE strategy based on optimal space filling design (Optimal Latin Hypercube DoEs) discussed by Kianifar et al [41], and implemented into a custom Matlab toolbox [42] was employed. This approach is based on a model building (MB) model validation (MV) sequence, where a response surface model is fitted to the data from the MB OLH DoE, which is then validated using both internal and external validation criteria, using the MV DoE set.

The overall procedure for the SRM engine model sensitivity analysis includes the following steps:

1. Define the internal and external parameters to be included in the sensitivity study, and for each parameter the relevant ranges considered for the experiment;

2. Generate the MB-MV OLH DoEs with the parameters chosen;

3. Run the SRM engine model for every DoE run as combination of input parameters, and record as responses:

(i) the in-cylinder pressure trace modelling error (using the Root Mean Squared Error (RMSE) of the errors between the fitted and measured traces, evaluated on a CAD basis;

(ii) the predicted NOx emissions, in parts per million by volume (PPMV).

4. Fit a response surface model for the responses from the DoE;

5. Evaluate the quality of the models using appropriate statistical measures - e.g. PRESS RMSE and Validation RMSE [41];

6. If the model is not validated, further test points are needed, so return to step (2); 
7. Analyse and evaluate trends from the model - comparing against engineering judgement, and extract information for model calibration.

The detailed sensitivity analysis was carried out using the procedure described above, initially at one engine speed / load set point - highlighted with a circled diamond in Figure 2. The sensitivity study experiments were then repeated at several other set points (shown as "diamond" points in Figure 2) across the engine speed-load envelope. The results from the global sensitivity study are used to derive settings for the SRM model parameters that can be used across the engine operating range. Given that the objectives of the SRM engine model calibration is both to minimize in-cylinder pressure errors and NOx prediction errors, finding the global set of parameters will be treated as a multi-objective optimization problem.

\section{Results: SRM Engine Model Sensitivity Study}

The following sections present the results of the study, based on the Diesel engine case study and the methodology outlined in the previous section.

\section{Sensitivity Study of SRM Engine Model for Diesel NOx Emissions}

In order to study the sensitivity of the SRM engine model simulation results to the input parameters, a set of separate DOEs were planned and run for the SRM internal and external parameters, respectively. In order to validate the procedure, the detailed sensitivity study was carried out at one speed load point, highlighted with a red circle in Figure 2.

Figure 6 illustrates the correlation between the measured and predicted in-cylinder pressure traces and derived AHRR profiles, based on the initial set up of the SRM engine model. The set of external and internal parameters used for the DOE experiments are summarised in Table 4 and Table 5, respectively. For the external parameters, apart from the iEGR parameter, the limits for the sensitivity study experiments were set at $\pm 10 \%$ from the nominal reading for this speed-load point. For the iEGR, the limits were set in conjunction with the experts based on expected range, underpinned by the analysis of the 1D simulation results shown in Figure 3. For the internal parameters, the ranges were defined based on the preliminary tests of SRM modelling.

Each of the DOEs consisted of a space filling OLH design with 70 test points, and additional 15 validation points, generated using the Model-Building - Model Validation procedure described in [41] and using the custom Matlab toolbox described in [42]. The DOE design is illustrated in Figure 5 as a 3D projection. The correlation between the predicted and measured in-cylinder pressure traces was considered in terms of the RMSE (root mean squared error) of the errors between the 2 traces (sum of squared errors calculated as in equation 9 , for the range between -60 to $+60 \mathrm{CAD}$ from TDC, as this is the part of the engine cycle where most of the combustion occurs, experimental pressure signal is stable and no noise is present). 


$$
R M S E=\sqrt{\frac{\sum_{i=-60}^{+60}(y(i)-b(i))^{2}}{\frac{120}{i}}}
$$

where $\boldsymbol{y}$ is a DOE point pressure profile, $\boldsymbol{b}$ is the experimental pressure profile, and $\boldsymbol{i}$ is the CAD step.

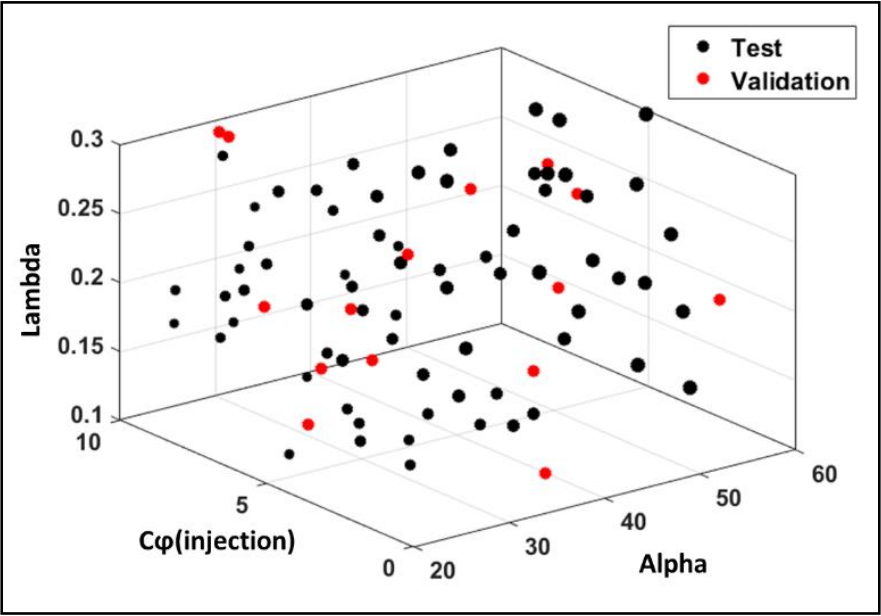

Figure 5: Visualisation of OLH DOE design.

In order to facilitate the analysis and visualization of the results, a Gaussian Process Model (Kriging) response surface model was fitted for both NOx and in-cylinder pressure trace error RMSE.
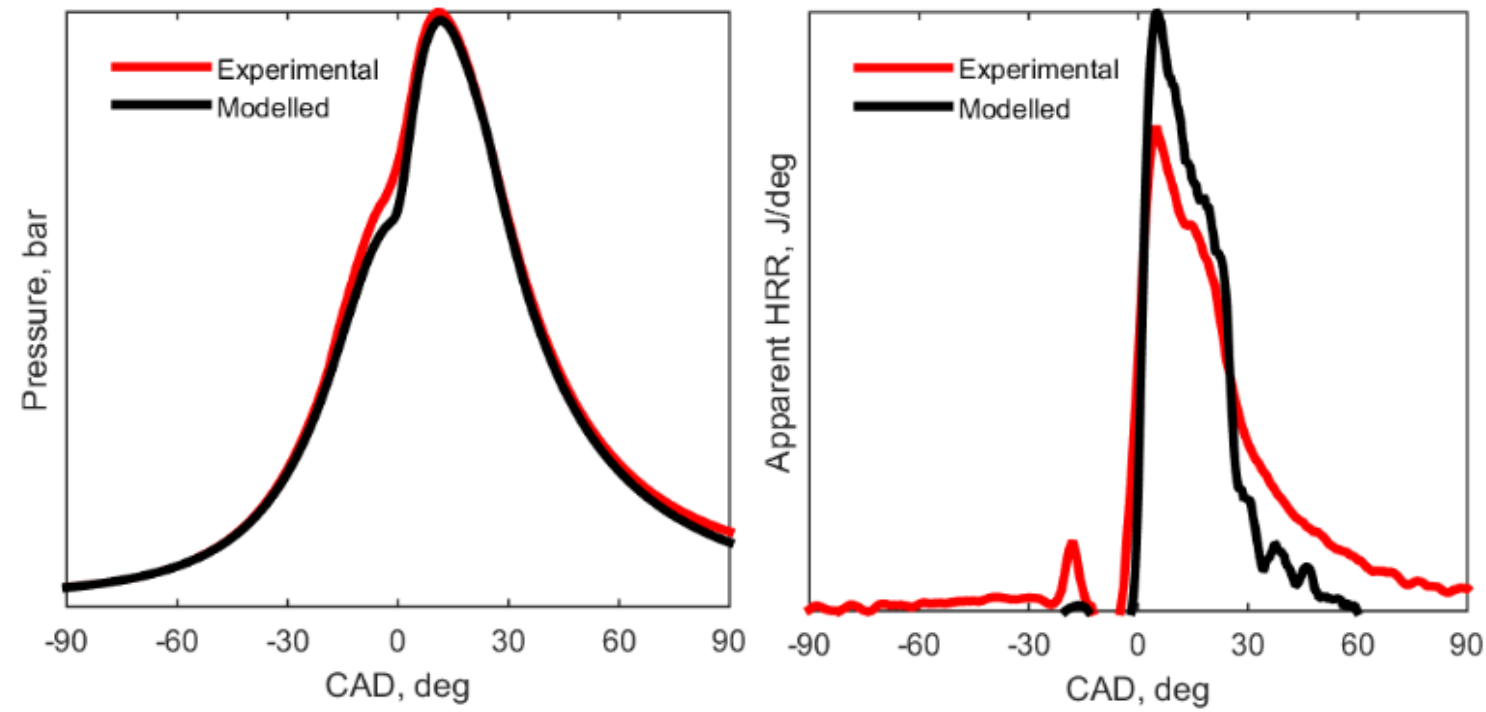

Figure 6: In-cylinder pressure trace correlation between experimental and simulation results. 
Table 4: SRM model external parameters for sensitivity study.

\begin{tabular}{|l|c|}
\hline External Parameter & Range [Min - Max ] \\
\hline Inlet Pressure (IVC) -P(inl) & $2.98-3.64$ bar \\
\hline Inlet Temperature (IVC) - T(inl) & $360-440 \mathrm{~K}$ \\
\hline Internal EGR - iEGR & $0.01-0.05 \mathrm{MF}$ \\
\hline Injection Fuel Pressure - P(inj) & $1305-1595$ bar \\
\hline Injection Fuel Temperature - T(inj) & $296-362 \mathrm{~K}$ \\
\hline Piston Wall Temperature - T(wall) & $495-605 \mathrm{~K}$ \\
\hline
\end{tabular}

Table 5: SRM model internal model parameters for sensitivity study.

\begin{tabular}{|l|c|}
\hline Internal Parameter & Range [Min - Max] \\
\hline Evap. Constant $(\lambda)$ - Lambda & $0.1-0.3$ \\
\hline $\boldsymbol{C}_{\boldsymbol{\varphi}}$ & $1-6$ \\
\hline $\mathbf{C}_{\boldsymbol{\varphi}(\text { injection })}$ & $2-10$ \\
\hline Injection Alpha & $20-60$ \\
\hline EOI Lag & $0-4$ CAD \\
\hline Woschni C1 & $1-10$ \\
\hline
\end{tabular}

\section{Analysis of the Effect of SRM External Parameters}

Figure 7 summarizes the NOx prediction models, showed as a pseudo-main effect plot - as a centre axis projection against each of the DoE parameters, extracted from the fitted response surface model. The fitted NOx model had good statistical properties, with both internal validation (PRESS RMSE) and external validation (Validation RMSE) smaller than $3.75 \%$ of the mean response (actual values - RESS RMSE - 43ppmv; Validation RMSE - 63ppmv). Figure 8 summarizes the analysis of the RMSE of the in-cylinder pressure trace error (as a statistical measure of the modelling error), as a pseudo-main effect of the SRM external parameters. This shows good statistical properties for the fitted model (PRESS RMSE 0.32 and validation RMSE 0.36).

The analysis of the results in Figures 7 and 8 in terms of the effect of each external parameter, can be summarized as follows:

Inlet Pressure $\{\boldsymbol{P}(\boldsymbol{i n l})\}$ - has a significant effect on the NOx result (Figure 7); as the inlet pressure increases, the NOx also increases drastically. This is consistent with cycle pattern, as higher inlet pressure increases the density of the air at the inlet and hence for the same volume allows to draw more air mass in the cylinder, resulting in more vigorous combustion, higher in-cylinder pressures and temperatures, leading to higher NOx. It is also seen that inlet pressure has a significant nonlinear effect on in-cylinder pressure trace modelling accuracy (Figure 8), with higher errors seen at lower inlet pressure.

Inlet Temperature $\{\boldsymbol{T}(\boldsymbol{i n l})\}$ - has a significant effect on NOx, which is consistent with cycle trends: increasing the temperature at the inlet implies a higher combustion temperature overall, which results in higher NOx. However, as the temperature increases, 
the density of the air decreases, which results in the lower amount of air mass trapped in the cylinder, and hence less vigorous combustion. This counters the effect gained from the in-cylinder temperature increase, and therefore the NOx response change is not as large as for the inlet pressure. The effect on the pressure trace modelling accuracy is also significant - as Figure 7 shows that higher inlet temperature values result in higher RMSE - so less accurate pressure trace matching.

Internal EGR (iEGR) - given that the SRM model makes no difference between internal and external EGR, this analysis accounts the total EGR defined in SRM as the part of the overall EGR mass fraction. Figure 7 shows the impact of EGR, within the set range for the experiment, on predicted NOx. The modelled response shows a logical pattern - as the EGR increases, the NOx is reduced, which is essentially the purpose of the EGR. Quantification of this effect (as shown in Figure 7) has significant practical importance in relation to engine modelling, since, as discussed previously, it is not possible to actually measure the exhaust gas residuals in the cylinder for correlation / calibration / validation purposes. Consequently, errors in the EGR input into the SRM model will have a very significant impact onto the NOx predictions. In terms of the effect on modelled pressure trace RMSE Figure 8 shows that EGR has relatively small / negligible effect.

Injection Fuel Pressure $\{\boldsymbol{P}($ inj $)\}$ - has little effect on both NOx and pressure trace modelling error. Fuel pressure is used in SRM to account for the Sauter Mean Diameter, which becomes less with the pressure increase, i.e. better atomisation occurs, faster evaporation and mixing, explaining the very slight NOx increase seen in Figure 7.

Injection Fuel Temperature $\{$ T(inj)\} - appears to have a negligible influence on both responses.

Piston Wall Temperature $\{\boldsymbol{T}($ wall $)\}$ - does not have significant effect on the NOx response. While there is heat transfer between the piston crown and the air/fuel mixture, as well as the time the mixture spends at the temperature that is less than that of the piston crown, this is not enough to affect the overall combustion temperature much. The piston wall temperature parameter - within the range considered for the DoE has a negligible effect on the pressure trace RMSE. 


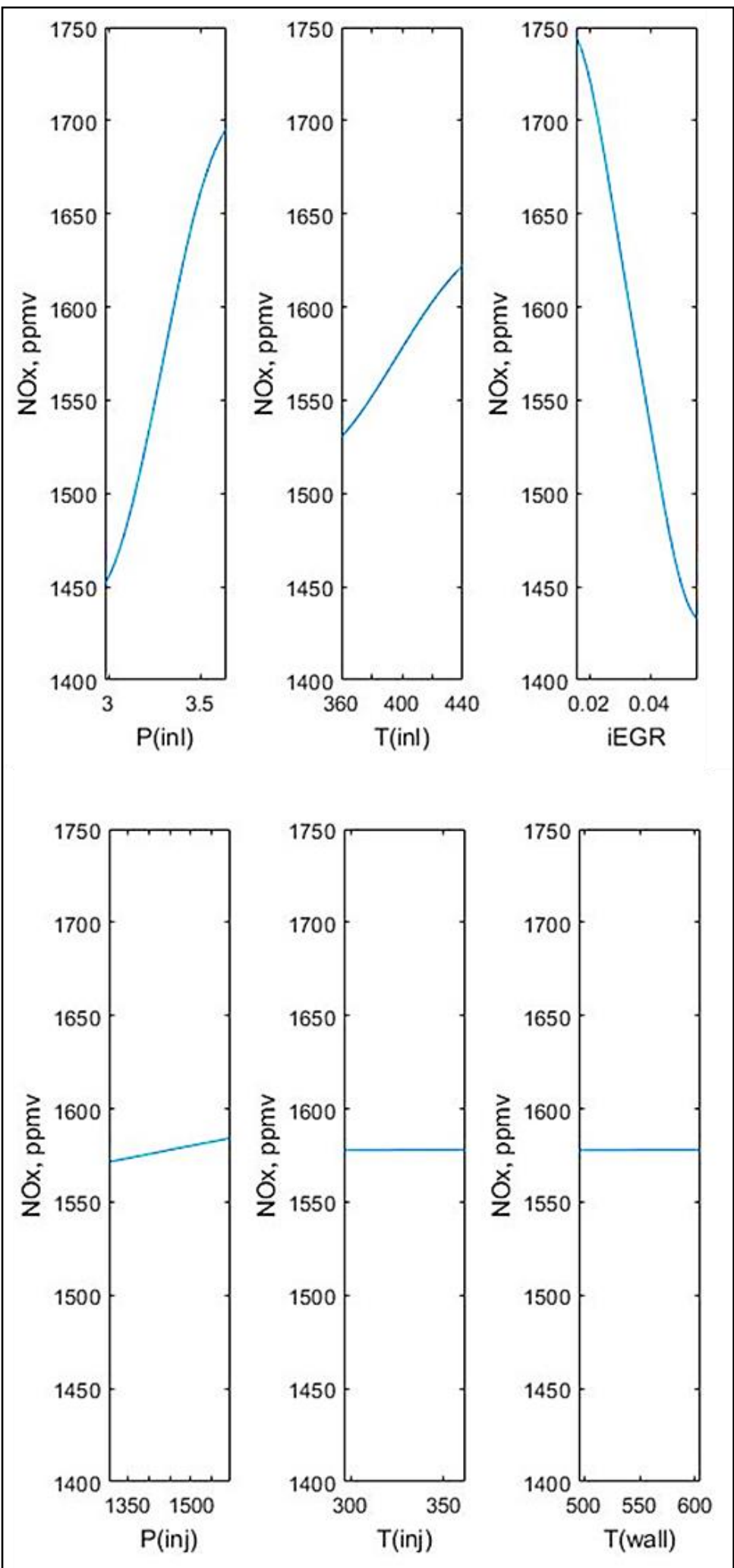

Figure 7: Sensitivity analysis: SRM External Parameters effects on predicted NOx emissions. 


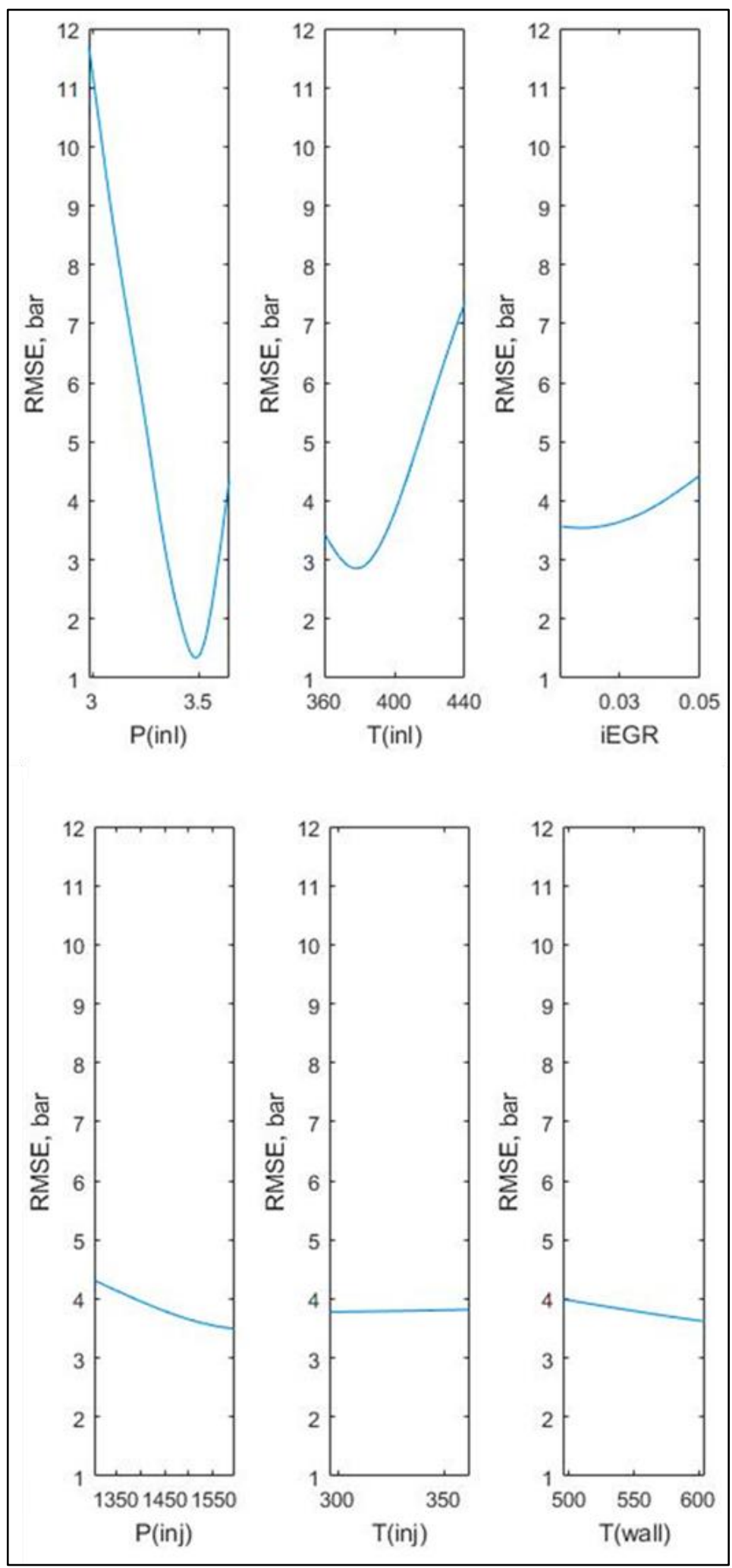

Figure 8: Sensitivity analysis: SRM External Parameters effects on in-cylinder pressure trace error / RMSE. 
Figure 9 summarizes fitted NOx prediction model as a function of SRM internal / tuning parameters, shown as pseudo-main effects plot (centre axis projections) against each parameter, extracted from the fitted response surface model. The fitted NOx model shows reasonable statistical properties (PRESS RMSE - 171ppmv; Validation RMSE 108ppmv). Figure 10 summarizes the RMSE of the modelled in-cylinder pressure trace error as pseudo-main effects against each internal parameter. The fitted model shows reasonably consistent statistical properties (PRESS RMSE 1.7 and validation RMSE 1.2).

Evaporation Constant $(\lambda)$ - has an increasing effect on the predicted NOx, which conforms with the engineering expectation. As the higher values of $\lambda$ denote higher evaporation rates, this means that more fuel is available in the gas state at the beginning of the combustion, which makes the combustion more vigorous and leads to higher NOx emissions. It is also seen from Figure 10 that $\lambda$ has a nonlinear effect on the in-cylinder pressure trace, with lower levels of $\lambda$ associated with bigger RMSE errors in pressure trace matching.

$\boldsymbol{C} \boldsymbol{\varphi}$-does not appear to have a significant effect on either NOx or the in-cylinder pressure trace modelling accuracy.

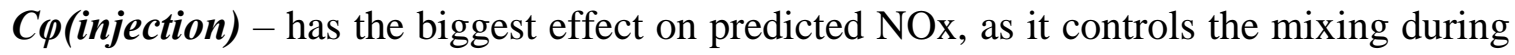
the injection time, which also accommodates the start of the combustion. Hence, with higher values of $\boldsymbol{C} \boldsymbol{\varphi}$ parameter the in-cylinder mixture is more homogeneous due to better mixing, and the combustion happens quicker, which results in higher NOx. $\boldsymbol{C} \boldsymbol{\varphi}$ has a large non-linear effect on the pressure trace - with both low and high values being associated with higher RMSE modelling errors for the pressure trace.

Injection Alpha - shows a small effect on both NOx and pressure trace RMSE. As the incylinder mixture becomes more stratified, inhomogeneous (higher Alpha), the NOx starts to decrease due to decreased combustion quality, which explains the decreasing effect on NOx. 

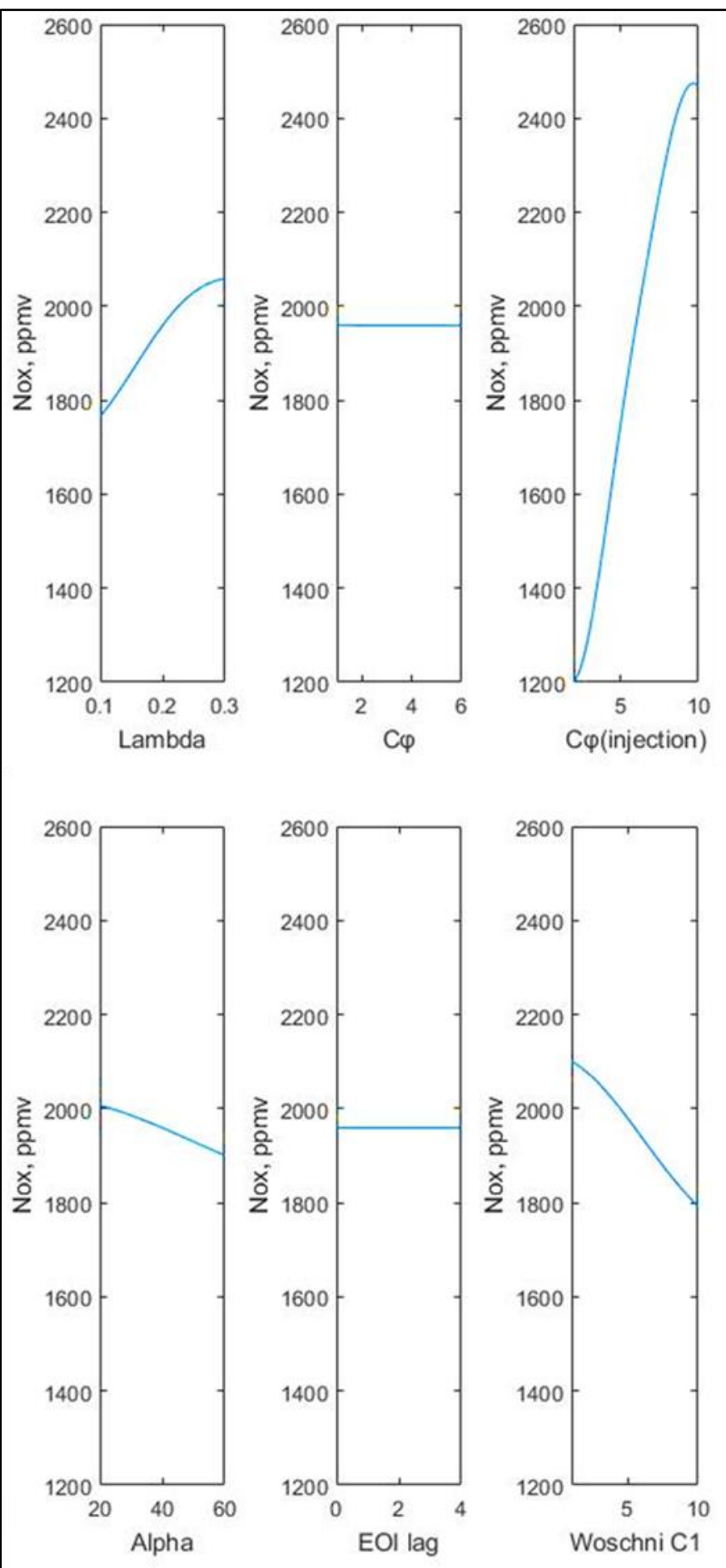

Figure 9: Sensitivity analysis: SRM Internal Parameters effects on predicted NOx emissions. 

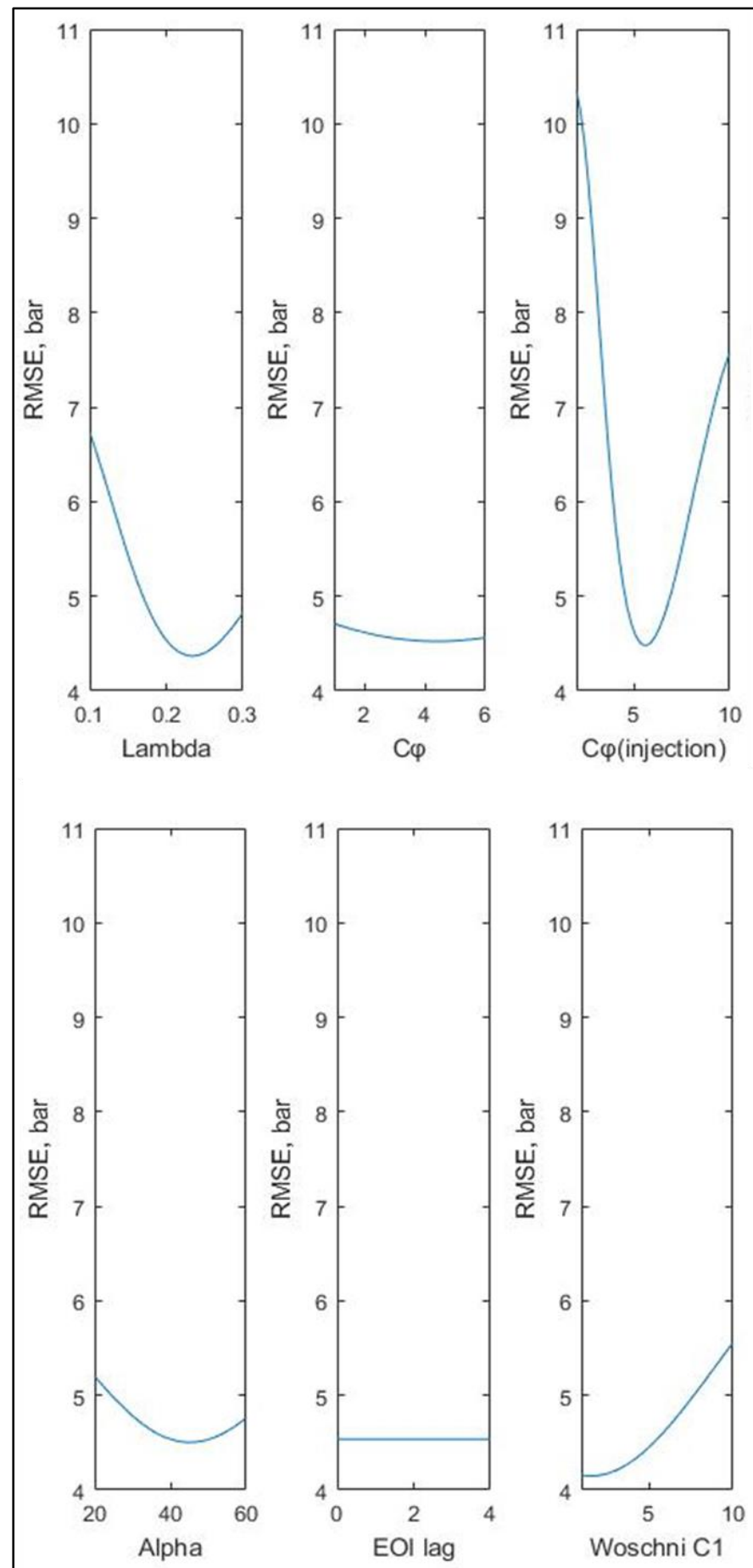

Figure 10: Sensitivity analysis: SRM Internal Parameters effects on in-cylinder pressure trace error / RMSE.

EOI Lag - within the experimental range for the DoE does not have any effect on either NOx or the pressure trace RMSE. This is likely due to the fact the main part of the combustion happens within the region of the injection itself, and the experimental window of just few CA degrees to prolong the period does not produce a significant difference. 
Woschni $\boldsymbol{C 1}$ - the results in Figure 9 show a small decreasing effect on NOx which it also confirms engineering expectations: an increase in the $\mathrm{C} 1$ Woschni correlation constant, this corresponds to an increased heat rejection to the walls, and therefore a decrease in the overall in-cylinder temperature, which decreases the amount of NOx produced. Figure 10 suggests a relatively small increasing effect on the in-cylinder pressure trace RMSE.

\section{SRM Model Calibration}

The detailed sensitivity study presented in the previous section has provided a useful insight on the effect of modelling parameters - both internal to the SRM modelling package, and the external inputs to the model. The model "calibration" task in this context is related to the choice of a set of values for the internal modelling parameters such that a good correlation is achieved with the measured data collected from engine experiments. For the specific test point studied, this would consist of selecting settings for the internal SRM variables that give the best correlation with the measured in-cylinder pressure traces, e.g. minimizing RMSE based on the fitted response surface model.

From a practical application point of view, the SRM engine model is expected to produce predictions at all engine speed-load points and running conditions across relevant to the drive cycle considered. This raises a significant challenge of developing a calibration for the SRM engine model that would ensure good fidelity across the engine operating range. While adjusting the calibration parameters for each speed-load point would give a potential benefit in fidelity, this would be an impractical strategy. Having one set for the whole domain is beneficial in terms of time expense and complexity associated with the model building for a particular engine or application.

In order to identify an optimal set of calibration parameters for the whole engine operating domain, the strategy adopted for this research was to:

(i) repeat the sensitivity study for a number of test points distributed in the operating domain;

(ii) derive a global optimal SRM calibration setting, optimal across the points where the sensitivity was studied in detail.

Therefore, a sub-set of 10 speed-load points were chosen from the 23 test points tested; these points are highlighted graphically (diamond marker) in Figure 2, and have been selected to ensure a reasonable coverage of the domain (the set includes the point for which the detailed sensitivity analysis was presented in the previous section). For each of the selected point, a characterization DOE experiment was run following the methodology described in the previous section. Thus, the DOE design for the SRM internal parameters sensitivity study (consisting of 70 model building and 15 validation points, as shown in Figure 5) was replicated for each of the additional 9 points. For each of the DOE points, the in-cylinder pressure trace RMSE (calculated using equation 8) was recorded as a response, as well as the predicted NOx, and response surface model were fitted and validated - using the methodology and process described for the sensitivity study. The 
detailed analysis of the models showed similar performance and validation to the one described for the initial test point discussed in details in the previous section.

A further important consideration for the SRM model calibration is that good performance in relation to NOx prediction is also desirable. The sensitivity study has shown that NOx predictions from the SRM model are also sensitive to the choice of internal parameters. By examining the trends shown in Figures 9 and 10 for the detailed sensitivity study, it is clear that there would be opportunities to consider a trade-off strategy for the selection of the optimal SRM model calibration. For example, choosing a value for the C $\varphi$ (injection) coefficient between 4.5 and 6.5 would make an insignificant difference to the fidelity of in-cylinder pressure trace matching (in terms of the RMSE between fitted and measured values, Figure 10); however, Figure 9 suggests that there is more than $40 \%$ variation in NOx prediction corresponding to this interval. Therefore, choosing a value for $\mathrm{C} \varphi$ (injection) that would deliver good fidelity for the pressure trace matching while minimizing the NOx prediction error would appear as a desirable strategy.

In order to exploit such opportunities in a systematic way, the approach put forward in this research was to carry out a global multi-objective trade-off optimization. The Matlab Model Based Calibration toolbox, which is commonly used for base engine calibration trade-off optimization, was employed for this study. The trade-off optimization approach is global as it was carried out across the 10 points where detailed sensitivity analysis against the SRM model internal parameters was available - with fitted models. The trade-off optimization seeks to find a set of SRM model internal parameters $(\boldsymbol{\theta})$ such that the overall in-cylinder pressure trace RMSE (defined as the sum of RMSEs of all 10 points studied, equation 9) and the overall NOx absolute error (defined as the sum of NOx absolute errors across the 10 points, equation 10) is minimised.

$$
\begin{aligned}
& \operatorname{RMSE}(\theta)=\sum_{i=1}^{10} \operatorname{RMSE}_{i}(\theta) \\
& \left.\Delta N O x(\theta)=\sum_{i=1}^{10} \mid N O x_{i}(\theta)-N O x_{i}^{T e s t}\right) \mid
\end{aligned}
$$

Where $N O x_{i}^{\text {Test }}$ is the measured NOx in engine test at set point $i ; N O x_{i}(\theta)$ is the predicted NOx from the fitted response surface model; and $\Delta N O x(\theta)$ is the overall absolute NOx error. The multi-objective Normal Boundary Intersection optimisation algorithm available in the MBC Matlab toolbox was applied to find a trade-off optimal solution with $R M S E(\theta)$ and $\Delta N O x(\theta)$ as objectives, as shown in equation 11 . The results of the optimisation are presented as a Pareto trade-off frontier in Figure 11.

$$
\min _{\theta}\{R M S E(\theta) ; \Delta N O x(\theta)\}
$$




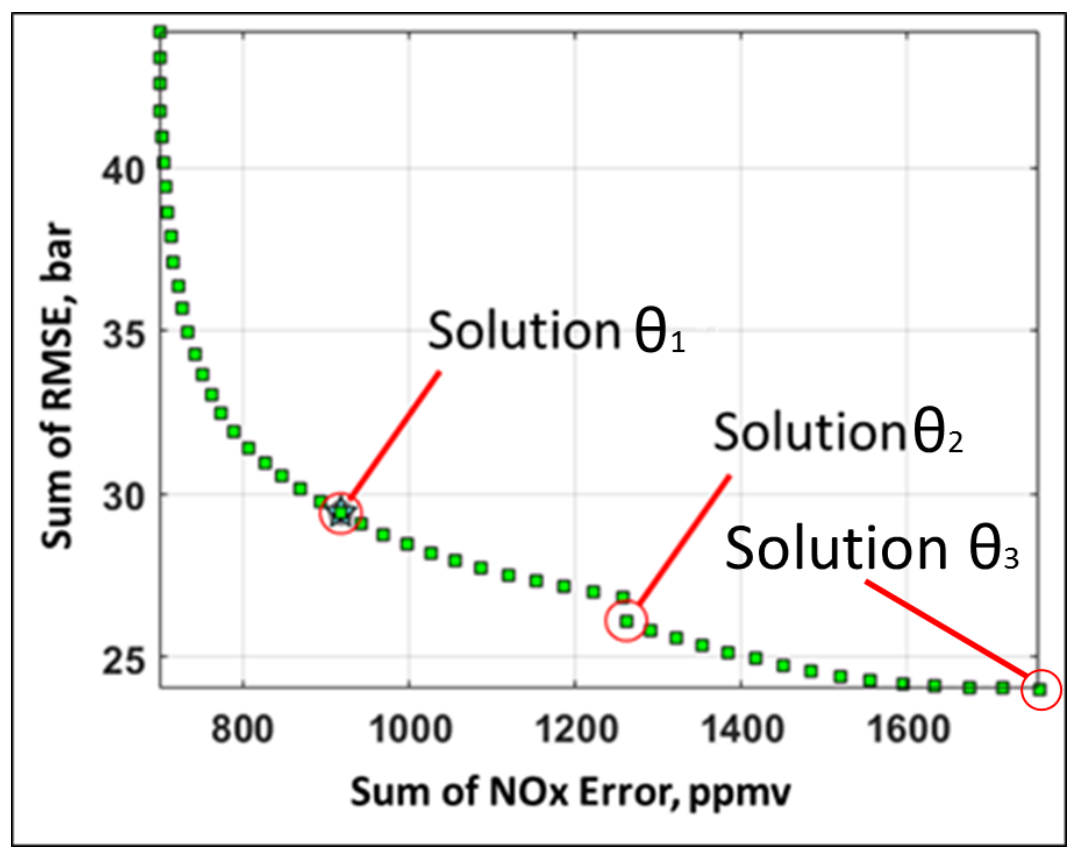

Figure 11: Trade-off between RMSE sum and NOx absolute error sum for 10 calibration points.

The benefits of the trade-off analysis are illustrated in Figure 11 by considering 3 solutions - $\theta_{1}, \theta_{2}$ and $\theta_{3}$, with parameter values detailed Table 4 . This analysis shows that a significant improvement in NOx prediction error can be achieved by optimal tuning of the SRM model internal parameters, without significant detrimental effect on the accuracy of the model validation (in relation to matching the in-cylinder pressure traces). For example, moving from solution $\theta_{3}$ to solution $\theta_{2}$ provides an improvement of over $30 \%$ in NOx prediction error, whilst the overall RMSE error gain is less than $8 \%$. Solution $\theta_{1}$ provides further improvement in NOx will limited increase in RMSE error. Table 4 shows the effect

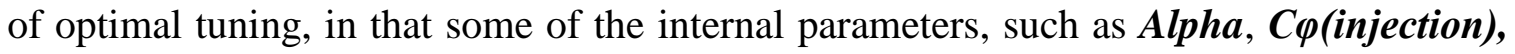
Evaporation Constant and Woschni $\boldsymbol{C 1}$ are significantly different between the $\theta_{2}$ and $\theta_{1}$ solutions illustrated. Importantly, this can be explained in relation to the physical effects associated with these internal parameters, discussed within the sensitivity analysis section. There is a clear interaction between these effects which act differently in relation to different responses, which explains the compromise between $\triangle$ NOx and RMSE errors. Solution $\theta_{1}$ was chosen as the $\Delta \mathrm{NOx}$ error sum is reduced significantly, while the overall RMSE error is still low.

Table 6. SRM Optimal Parameters Corresponding to $\boldsymbol{\Theta}_{1}, \boldsymbol{\theta}_{2}$ and $\boldsymbol{\Theta}_{3}$.

$\begin{array}{cccc} & \text { Solution } \theta_{1} & \text { Solution } \theta_{2} & \text { Solution } \theta_{3} \\ \text { Injection Alpha } & 60 & 33.22 & 24.45 \\ \text { C } \varphi & 3.024 & 3.016 & 2.872 \\ \text { C } \varphi \text { (injection) } & 6.095 & 4.395 & 4.676 \\ \text { EOI Lag } & 1.36 & 2.43 & 3.01 \\ \text { Evaporation Constant }(\lambda) & 0.207 & 0.162 & 0.161\end{array}$


$\begin{array}{llll}\text { Woschni Cl } & 8.786 & 2.387 & 1.000\end{array}$

\section{SRM Model Validation: Analysis of In-Cylinder Conditions Correlation}

In order to validate the capability of the tuned SRM model, a simulation analysis was carried out across the 23 speed-load test points (Figure 2), covering the whole engine operating domain. The simulation setup employed the parameters obtained through the automatic calibration methodology described in previous section and presented in Figure 11 and Table 4.

Overall, as can be seen in Figure 12, the predicted in-cylinder pressure traces match the experimental results reasonably well. The compression stage, up until the first pilot injection, is predicted very well. Peak pressure predictions, illustrated in Figure 13, are good, along with the auto-ignition timing. In most cases, in general across the low/medium load regions, the apparent Heat Release Rate (aHRR) profiles are also predicted well. However, some issues have been identified with aHRR profiles at some speed-load points where it was noted that aHRR rises from the main injection slightly asynchronously with the experimental data. It was also observed that the model does not perform very well in relation to pilot injections; this can be seen in Figure 12, where the model appears unable to reliably identify the aHRR from the pilot injection, which essentially means that the pilot injection does not auto-ignite as quickly as it is required. The latter issue has an effect on the combustion speed, which can be judged by examining Figure 14 where the crankshaft angle degrees (CAD) aTDC at 50\% mass fraction burned is presented for the experimental and modelled data, showing that simulated combustion is delayed in comparison to experimental at most of the speed-load points. However, the general trends are captured correctly and the modelled results closely resemble the experimental data.

\section{SRM Model Validation: Prediction of NOx Emissions}

Figure 15 and Figure 16 illustrate the validation of simulated NOx results against the experimental data. The results are normalized by the highest experimental NOx value, as per equation 13.

$$
\text { NOx }(\text { normalised })=\frac{\text { NOx }}{\max \{\text { NOx }(\exp )\}}
$$

Where $\max \{\boldsymbol{N O x}(\boldsymbol{e x p})\}$ is the highest experimental NOx measurement.

Figures 15 and 16 show that the NOx prediction capabilities of the SRM engine model are satisfactory. Figure 15 shows that the trends are predicted correctly - the NOx emissions output increases with the engine load increase. The error, however, increases with the load increase. Looking at the aHRR profiles for the high load region, a significant peak aHRR overshooting from the model side can be noticed, which increases with the engine speed (likely related to increased turbulence). Considering that the issue is not seen in lower load and lower speed regions, this might provide an argument for different values of calibration parameters across the domain; in particular the $\mathrm{C} \varphi$ (injection) and $\mathrm{C} \varphi$ that account for the turbulence. However, it is also possible that the injection rate profiles have an influence, 
as they have a significant impact on the aHRR and combustion as such. The injector model lack of fidelity would manifest through: (i) inaccurate fuel mass split to the pilot injection; and (ii) incorrect fuel injection rate distribution throughout the main injection event. This way, the unburned pilot ignites just before or at the start of the main injection, also promoting the ignition of the freshly injected excessive main portion, which leads to more vigorous combustion, higher temperatures and as a result - overestimated NOx prediction. 


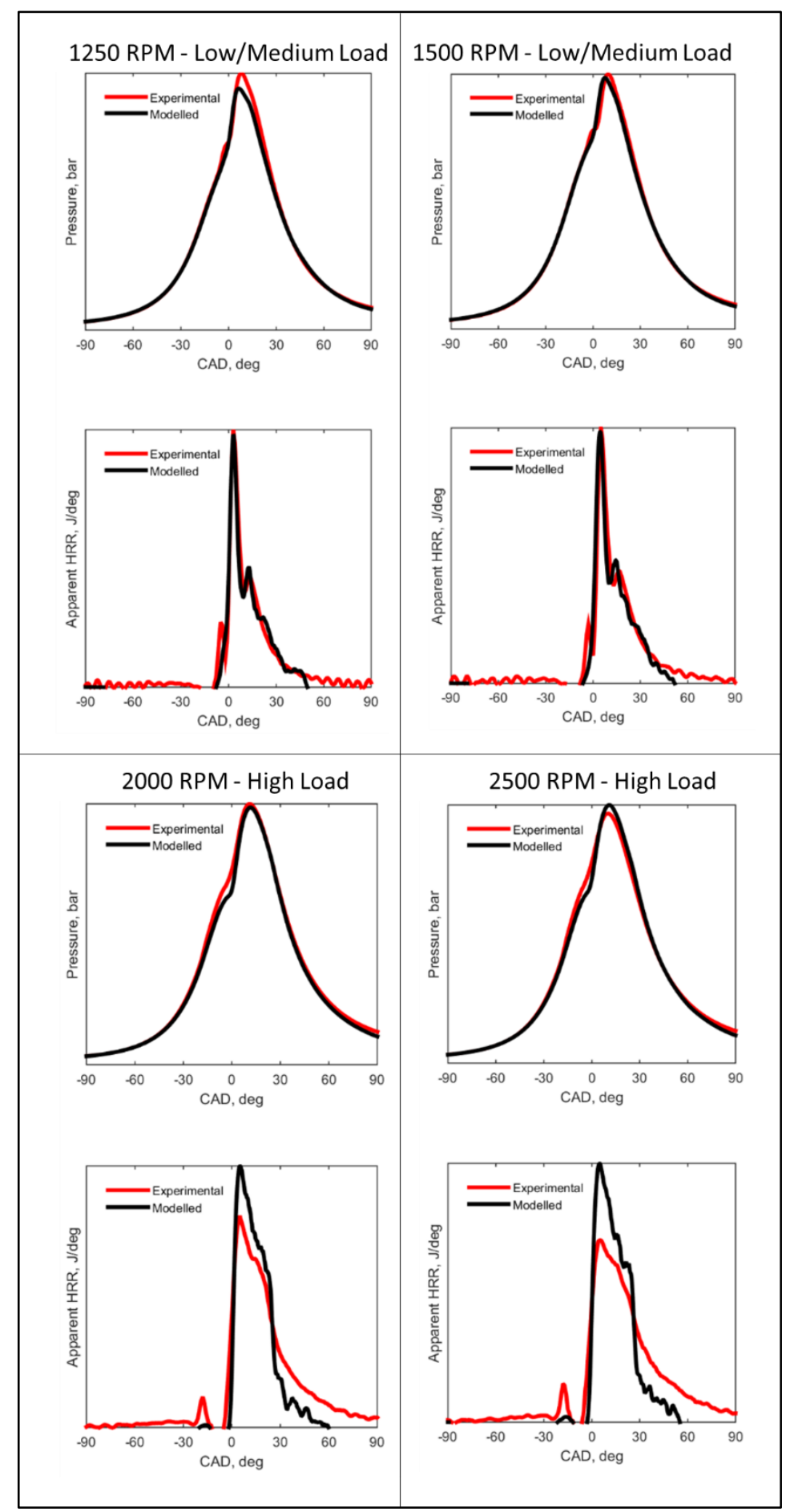

Figure 12: Analysis of in-cylinder conditions correlation. 


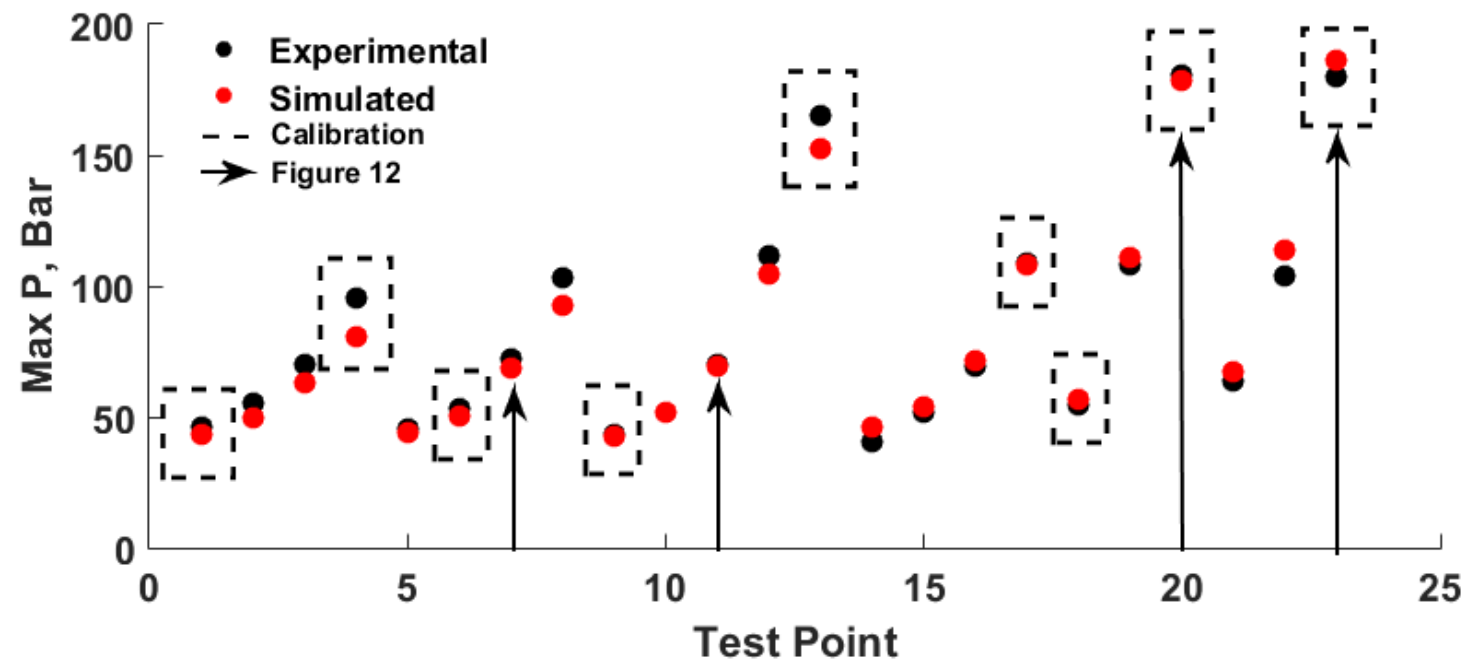

Figure 13: Maximum in-cylinder pressure - experimental and simulated (speed and load increases to the right).

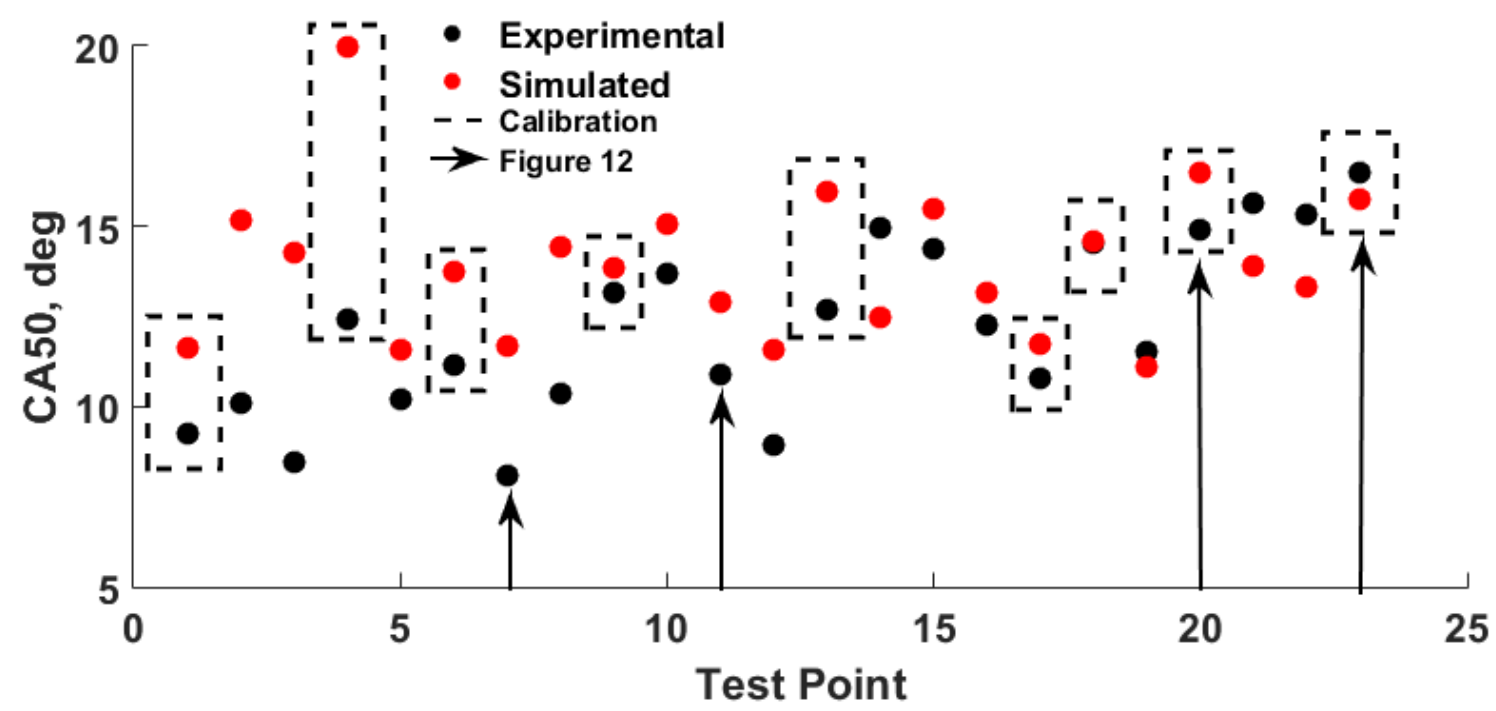

Figure 14: CAD aTDC at 50\% mass fraction burned - experimental and simulated (speed and load increases to the right). 


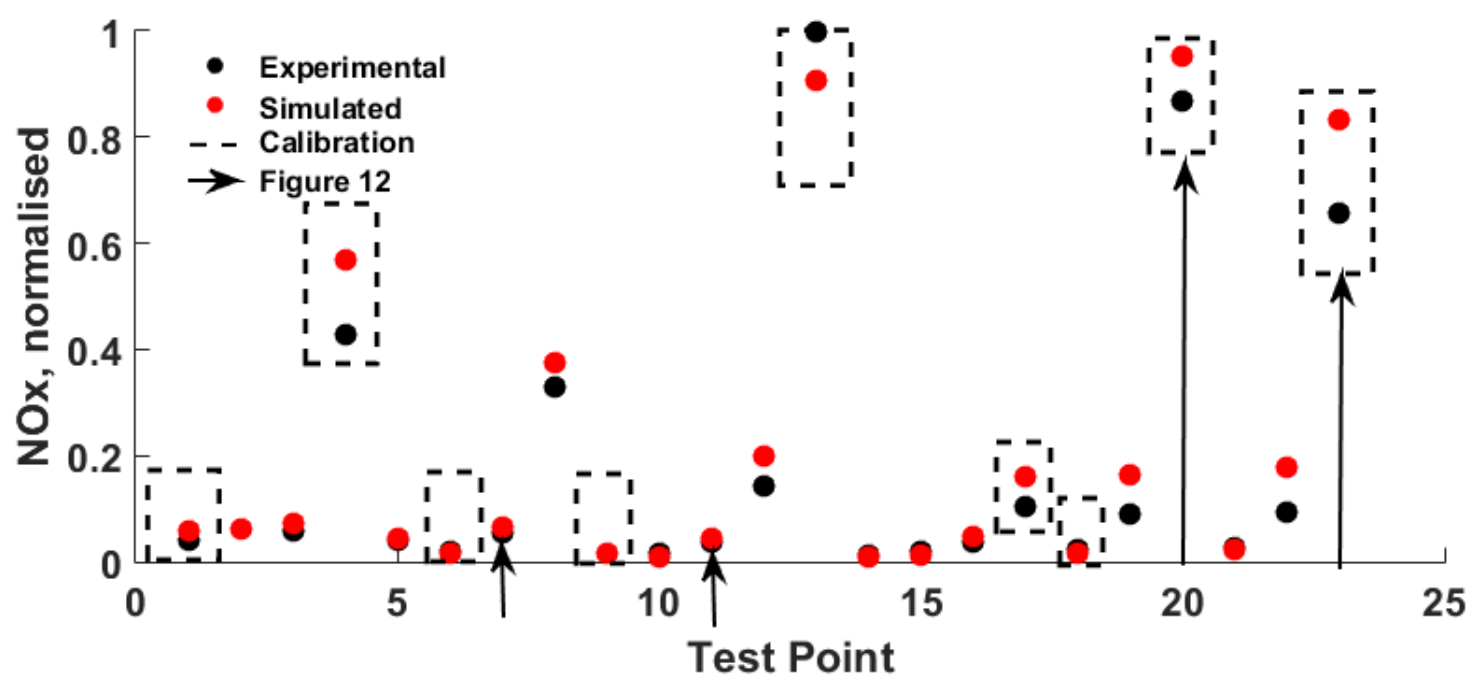

Figure 15: SRM NOx prediction - experimental and simulated (speed and load increases to the right).

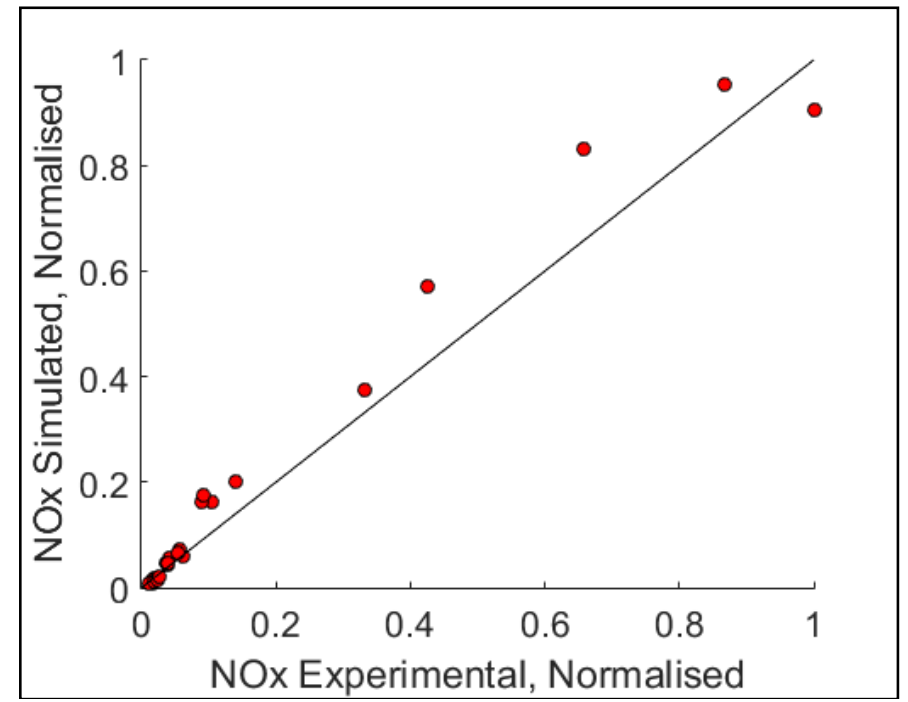

Figure 16: Normalised NOx simulated versus experimental results.

\section{Conclusions}

The systematic approach to the study of the performance of the CMCL SRM model for a Diesel engine application described in this paper, has enabled a rich analysis and discussion on the effects of parameters on the overall modelling performance, which are of significant usefulness and impact for its practical application.

The results of the sensitivity study in relation to the external parameters have shown that the SRM model adequately reacts to the external parameters changes. An important observation from the study is the sensitivity of the model to the EGR input, as $0.01 \mathrm{mf}$ EGR change leads to about 5\% NOx results difference. Considering, that the external EGR 
mass fraction measurement tolerance is $\pm 1 \%(0.01 \mathrm{mf})$, this alone can introduce a $10 \%$ error in the NOx prediction capability. Moreover, as discussed in the paper, it is not possible to precisely measure or estimate the amount of the exhaust gas residuals, which contribute to the total EGR, therefore this makes the NOx prediction fidelity even more challenging. While the trends are correctly predicted by the SRM model, which makes it useful for early powertrain development and calibration studies, given the uncertainty in the external SRM model parameters (as inputs), the accuracy of the NOx engine out emissions prediction is currently insufficient to support detailed work on other parts of the system (e.g. aftertreatment system control).

The paper makes important methodological contributions for the validation and tuning of the SRM engine model, which can have broad application impact for related studies. The proposed procedure for selecting the median cycle, described in detail in the paper, and used as the basis for evaluation of cycle modelling accuracy, provides a robust basis for validating engine / combustion simulation models against measured in-cylinder pressure traces. The sensitivity study on the SRM internal model parameters, coupled with the multiobjective trade-off optimization provides and effective strategy for SRM model parameters selection. This can be regarded as an automatic calibration methodology, which enabled the selection of an optimal set of internal SRM parameters satisfying the modelling accuracy requirements across the engine operating domain. The multi-objective optimization methodology can easily extend to a much wider set of objectives to include performance metrics for other gaseous emissions (in particular soot / Pn, THC and CO). This would require detailed sensitivity studies for the internal SRM model parameters against all emissions, beyond the scope of the current study.

The validation results for the case study showed good in-cylinder conditions correlation across the engine speed / load range of interest. The pressure profiles closely resemble the profiles recorded experimentally. however, the model most of the time cannot capture the apparent heat release rate from the pilot injection event, which results in loss of fidelity of the correlation. As discussed, this could be linked to the challenge to replicate in simulation the exact injection rate profile as in the experiment, or current limitation with the software.

Overall, despite modelling imperfections which only serve to reinforce the fact that engine modelling and simulation is still a challenging field, the SRM modelling software has generally shown good capability for modelling Diesel engine combustion. CMCL SRM modelling environment is convenient for practical use, and provides reasonable simulation expense, requiring 2-3 minutes to run one engine cycle. The model predicts NOx emissions well, with trends captured very well and predicted values are close to the experiments. Considering the NOx sensitivity to EGR and uncertainty in the EGR experimental data, this is a good achievement.

\section{Funding}

The authors acknowledge funding for the research presented in this paper from Jaguar Land Rover under a research collaboration with the University of Bradford. 


\section{References:}

1. Bernard, G., Scaife, M., Bhave, A., Ooi, D. et al., "Application of the SRM Engine Suite over the Entire Load-Speed Operation of a U.S. EPA Tier 4 Capable IC Engine," SAE Technical Paper 2016-01-0571, 2016, doi:10.4271/201601-0571.

2. Smallbone, A., Bhave, A., Coble, A., Mosbach, S. et al., "Identifying Optimal Operating Points in Terms of Engineering Constraints and Regulated Emissions in Modern Diesel Engines," SAE Technical Paper 2011-01-1388, 2011, doi:10.4271/2011-01-1388.

3. Su, H., Mosbach, S., Kraft, M., Bhave, A. et al., "Two-stage Fuel Direct Injection in a Diesel Fuelled HCCI Engine," SAE Technical Paper 2007-01-1880, 2007, doi:10.4271/2007-01-1880.

4. Mosbach, S., Kraft, M., Bhave, A., Mauss, F. et al., "Simulating a Homogeneous Charge Compression Ignition Engine Fuelled with a DEE/EtOH Blend," SAE Technical Paper 2006-01-1362, 2006, doi:10.4271/2006-01-1362.

5. Mosbach, S., Su, H., Kraft, M., Bhave, A. et al., "Dual injection homogeneous charge compression ignition engine simulation using a stochastic reactor model," International Journal of Engine Research Vol 8, Issue 1, pp. 41 - 50, 2007, doi:10.1243/14680874JER01806.

6. Perlman, C., Frojd, K., Seidel, L., Tuner, M. et al., "A Fast Tool for Predictive IC Engine In-Cylinder Modelling with Detailed Chemistry," SAE Technical Paper 2012-01-1074, 2012, doi:10.4271/2012-01-1074.

7. Lundgren, M., Tuner, M., Johansson, B., Bjerkborn, S. et al., "Gasoline PPC: A Parametric Study of Late Cycle Mixing Conditions using a Predictive Two-zone SRM Modeling Tool," SAE Technical Paper 2013-01-2621, 2013, doi:10.4271/2013-01-2621.

8. Lucchini, T., D'Errico, G., Contino, F., and Jangi, M., "Towards the Use of Eulerian Field PDF Methods for Combustion Modeling in IC Engines," SAE Int. J. Engines 7(1):286-296, 2014, doi:10.4271/2014-01-1144.

9. Matrisciano, A., Borg, A., Perlman, C., Lehtiniemi, H. et al., "Soot Source Term Tabulation Strategy for Diesel Engine Simulations with SRM," SAE Technical Paper 2015-24-2400, 2015, doi:10.4271/2015-24-2400.

10. Chow A., and Wyszynski, M. L., "Thermodynamic modelling of complete engine systems - a review," Proceedings of the Institution of Mechanical Engineers, Part D: Journal of Automobile Engineering vol. 213, no. 4, pp. 403415, 1999, doi:10.1243/0954407991526964.

11. Dibble, R., Au, M., Girard, J., Aceves, S. et al., "Current Research in HCCI Combustion at UC Berkeley and LLNL," SAE Technical Paper 2001-01-2511, 2001, doi:10.4271/2001-01-2511.

12. Arsie, I., Di Genova, F., Pianese, C., Sorrentino, M. et al., "Development and Identification of Phenomenological Models for Combustion and Emissions of Common-Rail Multi-Jet Diesel Engines," SAE Technical Paper 2004-011877, 2004, doi:10.4271/2004-01-1877.

13. Zheng J., and Caton J. A., "Use of a single-zone thermodynamic model with detailed chemistry to study a natural gas fueled homogeneous charge compression ignition engine," Energy Conversion and Management, vol. 53, no. 1, pp. 298-304, 2012, doi:10.1016/j.enconman.2011.09.005.

14. Jia, M., Xie, M., and Peng, Z., "A Comparative Study of Multi-zone Combustion Models for HCCI Engines," SAE Technical Paper 2008-01-0064, 2008, doi:10.4271/2008-01-0064.

15. Hountalas, D., Lamaris, V., and Pariotis, E., "Identification of the Error Introduced in DI Diesel Engine Phenomenological Multi-Zone Models from Assumptions Related to the Initial Conditions at the Nozzle Exit," SAE Technical Paper 2010-01-0153, 2010, doi:10.4271/2010-01-0153.

16. Ogink, R. and Golovitchev, V., "Gasoline HCCI Modeling: An Engine Cycle Simulation Code with a Multi-Zone Combustion Model," SAE Technical Paper 2002-01-1745, 2002, doi: 10.4271/2002-01-1745.

17. Poetsch, C., Ofner, H., and Schutting, E., "Assessment of a Multi Zone Combustion Model for Analysis and Prediction of CI Engine Combustion and Emissions," SAE Technical Paper 2011-01-1439, 2011, doi:10.4271/201101-1439.

18. Bernard, G., Lebas, R., and Demoulin, F., "A OD Phenomenological Model Using Detailed Tabulated Chemistry Methods to Predict Diesel Combustion Heat Release and Pollutant Emissions," SAE Technical Paper 2011-01-0847, 2011, doi:10.4271/2011-01-0847.

19. Heywood J., "Internal combustion engine fundamentals, International Edition" (McGraw-Hill Book Company, 1988), p.749, ISBN: 0-07-100499-8

20. Masoudi, R., Adibi asl, H., Lashgarian Azad, N., and McPhee, J., "Parameter Identification of a Quasi-Dimensional Spark-Ignition Engine Combustion Model," SAE Technical Paper 2014-01-0385, 2014, doi:10.4271/2014-01-0385.

21. Mauviot, G., Albrecht, A., and Poinsot, T., "A New OD Approach for Diesel Combustion Modeling Coupling Probability Density Function with Complex Chemistry," SAE Technical Paper 2006-01-3332, 2006, doi: $10.4271 / 2006-01-3332$.

22. Bordet, N., Caillol, C., Higelin, P., and Talon, V., "A Physical OD Combustion Model Using Tabulated Chemistry with Presumed Probability Density Function Approach for Multi-Injection Diesel Engines," SAE Technical Paper 2010-01-1493, 2010, doi:10.4271/2010-01-1493. 
23. Pasternak, M., Mauss, F., and Bensler, H., "Diesel Engine Cycle Simulation with a Reduced Set of Modeling Parameters Based on Detailed Kinetics," SAE Technical Paper 2009-01-0676, 2009, doi:10.4271/2009-01-0676.

24. Franken, T. and Mauss, F., "Development of Methodology for Predictive Diesel Combustion Simulation Using 0D Stochastic Reactor Model," SAE Technical Paper 2016-01-0566, 2016, doi:10.4271/2016-01-0566.

25. Smallbone, A., Bhave, A., Coble, A., Mosbach, S. et al., "Simulating PM Emissions and Combustion Stability in Gasoline/Diesel Fuelled Engines," SAE Technical Paper 2011-01-1184, 2011, doi:10.4271/2011-01-1184.

26. Pasternak, M., Mauss F., Sens M., Riess, M. et al., "Gasoline engine simulations using zero-dimensional spark ignition stochastic reactor model and three-dimensional computational fluid dynamics engine model," International Journal of Engine Research vol. 17, no. 1, pp. 76-85, 2016, doi:10.1177/1468087415599859.

27. Themi, V., "Multi-physics Co-simulation of Engine Combustion and Exhaust Aftertreatment System," Ph.D. thesis, Faculty of Engineering and Informatics, University of Bradford, Bradford, 2016.

28. Su, H., Vikhansky, A., Mosbach, S., Kraft, M. et al., "A computational study of an HCCI engine with direct injection during gas exchange," Combustion and Flame vol. 147, no. 1-2, pp. 118-132, 2006, doi:10.1016/j.combustflame.2006.07.005.

29. Bhave, A., Balthasar, M., Kraft, M., and Mauss, F., "Analysis of a natural gas fuelled homogeneous charge compression ignition engine with exhaust gas recirculation using a stochastic reactor model," International Journal of Engine Research pp. Vol 5, Issue 1, pp. 93 - 104, 2004, doi:10.1243/146808704772914273.

30. Aldawood, A., Mosbach, S., and Kraft, M., "HCCI Combustion Phasing Transient Control by Hydrogen-Rich Gas: Investigation Using a Fast Detailed-Chemistry Full-Cycle Model," SAE Technical Paper 2009-01-1134, 2009, doi:10.4271/2009-01-1134.

31. Mosbach, S., Aldawood, A. M., and Kraft, M., "Real-Time Evaluation of a Detailed Chemistry HCCI Engine Model Using a Tabulation Technique," Combustion Science and Technology vol. 180, Issue 7, pp. 1263-1277, 2008, doi:10.1080/00102200802049414.

32. Mosbach, S., Celnik, M. S., Raj, A., and Kraft, M., et al., "Towards a detailed soot model for internal combustion engines," Combustion and Flame vol. 156, Issue 6, pp. 1156-1165, 2009, doi:10.1016/j.combustflame.2009.01.003.

33. Rezaei, Reza, et al. "Phenomenological Modeling of Combustion and NOx Emissions Using Detailed Tabulated Chemistry Methods in Diesel Engines." International Journal of Engine Research, vol. 17, no. 8, Oct. 2016, pp. 846856, doi:10.1177/1468087415619302.

34. Pasternak, Michal, et al. "Diesel Engine Performance Mapping Using a Parametrized Mixing Time Model." International Journal of Engine Research, vol. 19, no. 2, Feb. 2018, pp. 202-213, doi:10.1177/1468087417718115.

35. Lai, J., Parry, O., Mosbach, S., Bhave, A. et al., "Evaluating Emissions in a Modern Compression Ignition Engine Using Multi-Dimensional PDF-Based Stochastic Simulations and Statistical Surrogate Generation," SAE Technical Paper 2018-01-1739, 2018, https://doi.org/10.4271/2018-01-1739.

36. M. M. Elkotb, "Fuel Atomization for Spray Modelling," Progress in Energy and Combustion Science vol. 8, Issue 1, pp. 61-91, 1982, doi:10.1016/0360-1285(82)90009-0.

37. CMCL Innovations, "Kinetics \& SRM Engine Suite Manual," 2016.

38. Parra, C. A. F., "Heat transfer investigations in a modern diesel engine," Ph.D. thesis, Department of Mechanical Engineering, University of Bath, Bath, 2008.

39. Abbaszadehmosayebi, G., " Diesel engine heat release analysis by using newly defined dimensionless parameters," Ph.D. thesis, School of Engineering and Design, Brunel University, 2014.

40. Roberts, P. J., "Fuel and Residual Effects in Spark Ignition and Homogeneous Charge Compression Ignition Engines," Ph.D. thesis, School of Mechanical Engineering, University of Leeds, Leeds, 2010.

41. Kianifar, M. R., Campean, F., and Wood, A., "Application of permutation genetic algorithm for sequential model building-model validation design of experiments," Soft Computing Vol 20, Issue 8, pp. 3023-3044, 2016, doi:10.1007/s00500-015-1929-5.

42. Kianifar, M. R., "Sequential DoE and Multidisciplinary Optimisation Tools for Engine Experiments", Technical Report, University of Bradford, 2015.

\section{List of Abbreviations}

\section{aHRR}

BMEP

CAD

CAE
Apparent Heat Release Rate

Brake Mean Effective Pressure

Crankshaft Angle Degrees

Computer-aided Engineering 


\begin{tabular}{|c|c|}
\hline CFD & Computational Fluid Dynamics \\
\hline $\mathrm{CO}$ & Carbon Oxide \\
\hline DoE & Design of Experiments \\
\hline EGR & Exhaust Gas Recirculation \\
\hline HCCI & Homogeneous Charge Compression Ignition \\
\hline iEGR & Internal Exhaust Gas Recirculation (residuals) \\
\hline IVC & Inlet Valve Close \\
\hline mf & Mass Fraction \\
\hline NOx & Mono-nitrogen oxides \\
\hline OLH & Optimal space-filling Latin Hypercube \\
\hline PDF & Probability Density Function \\
\hline PDF & Probability Density Function \\
\hline Pn & Particle Number \\
\hline RMSE & Root Mean Squared Error \\
\hline SMD & Sauter Mean Diameter \\
\hline SOI / EOI & Start / End of Injection \\
\hline SRM & Stochastic Reactor Model \\
\hline SSE & Sum of Squared Errors \\
\hline $\begin{array}{l}\text { TDC / } \\
\text { BDC }\end{array}$ & Top / Bottom Dead Centre \\
\hline THC & Total Hydrocarbons \\
\hline
\end{tabular}

